TRANSACTIONS OF THE

AMERICAN MATHEMATICAL SOCIETY

Volume 354, Number 4, Pages 1351-1371

S 0002-9947(01)02846-X

Article electronically published on November 21, 2001

\title{
MILNOR CLASSES OF LOCAL COMPLETE INTERSECTIONS
}

\author{
J.-P. BRASSELET, D. LEHMANN, J. SEADE, AND T. SUWA
}

\begin{abstract}
Let $V$ be a compact local complete intersection defined as the zero set of a section of a holomorphic vector bundle over the ambient space. For each connected component $S$ of the singular set $\operatorname{Sing}(V)$ of $V$, we define the Milnor class $\mu_{*}(V, S)$ in the homology of $S$. The difference between the SchwartzMacPherson class and the Fulton-Johnson class of $V$ is shown to be equal to the sum of $\mu_{*}(V, S)$ over the connected components $S$ of $\operatorname{Sing}(V)$. This is done by proving Poincaré-Hopf type theorems for these classes with respect to suitable tangent frames. The 0-degree component $\mu_{0}(V, S)$ coincides with the Milnor numbers already defined by various authors in particular situations. We also give an explicit formula for $\mu_{*}(V, S)$ when $S$ is a non-singular component and $V$ satisfies the Whitney condition along $S$.
\end{abstract}

In his original paper, Chern gave several equivalent definitions for characteristic classes of complex manifolds. In the case of singular varieties, one has different ways to generalize these, leading to classes which are not necessarily identical. Among them are the Schwartz-MacPherson class (abbreviated as SM class) Sc1, [Ma], BS] and the Fulton-Johnson class (abbreviated as FJ class) [FJ], [F. Each one of them is defined in a relevant context and has its own interest and advantage. One of the motivations of this work is to compare the SM and the FJ classes using geometric interpretations of these classes. We perform this comparison in the case of local complete intersections. In this case, it is well known that the FJ class coincides with the image, by the Poincaré homomorphism, of the Chern class of the virtual tangent bundle.

The Schwartz class of a compact analytic variety $V$ is originally defined using socalled radial frames. These are frames tangent to a Whitney stratification of $V$, they have isolated singularities on a suitable skeleton of an appropriate triangulation and they are pointing outwards of suitable neighborhoods of the strata. The Schwartz class satisfies a decomposition property: the Schwartz class of $V$ is the sum of the Schwartz class of the singular part of $V$ and of a relative class associated to the regular part. Also, the Schwartz classes are localized in a subset related to the singularities of the radial frame.

We prove that these two properties of Schwartz classes generalize in the case of frames which may not be radial and are possibly with non-isolated singularities (section 2). Also, the Fulton-Johnson class satisfies similar properties (section 4).

Received by the editors July 15, 2000 and, in revised form, December 1, 2000.

2000 Mathematics Subject Classification. Primary 57R20; Secondary 14C17, 14J17, 32S55, $58 \mathrm{~K} 45$.

(C)2001 American Mathematical Society 
In particular, we obtain localization theorems for SM and FJ classes in the spirit of the Poincaré-Hopf theorem.

Let us consider the situation of a local complete intersection $V$ in a complex manifold $M$, defined as the zero set of a section of a holomorphic vector bundle over $M$, and denote by $S$ a compact connected subset either of the regular part or the singular part of $V$. We define the Schwartz and virtual classes of a frame at $S$ and prove the localization theorems. We also prove that, for a non-singular component of the singular set, the Schwartz and virtual classes of frames can be computed in terms of Schwartz and virtual indices of vector fields (Theorems 5.7 and 5.10).

As mentioned above, one of the main purposes of this work is to compare the SM and the FJ classes. There are already results in this direction ( $\mathrm{A} 2$, [P], [PP1, [SS2], [Su1, see also Br2] for a survey). In particular, when the difference lies in dimension 0, it is expressed in terms of Milnor numbers. It is natural to call Milnor classes the difference between SM and FJ classes for higher degrees.

In fact, the "localization theorem" (Theorem 5.2) tells us that the Milnor classes are localized on the singular set $\operatorname{Sing}(V)$ of $V$. Hence the SM and FJ classes coincide in dimensions higher than the dimension of $\operatorname{Sing}(V)$. In a more precise way, for each connected component $S$ of $\operatorname{Sing}(V)$, we obtain integral homology classes $\mu_{i}(V, S)$, $0 \leq i \leq \operatorname{dim} S$, which measure the contribution of $S$ to the Milnor classes. We call these the Milnor classes of $V$ at $S$. The 0 -degree term is shown to coincide with the Milnor number, as defined in $[\mathrm{Mi}],[\mathrm{H}]$ when $\operatorname{dim} S=0$ and in $[\mathrm{P}$ when $\operatorname{dim} S>0$ and $V$ is a hypersurface (see section 6 ). Therefore, this 0 -degree Milnor class can be called a generalized Milnor number, extending for local complete intersections the definition given in $\underline{\mathrm{P}}$.

One important application of the previous results is a Lefschetz type theorem for Milnor classes (Corollary 5.13), which is proved under the assumption that $S$ is non-singular and $V$ satisfies the Whitney condition along $S$ (see Remark 5.14 for a weaker assumption).

It is well known that for isolated singularities the Milnor number is the number of vanishing cycles in a Milnor fiber; in this case all the other Milnor classes are automatically 0 . It would be interesting to see whether there is a similar statement when the singular set has dimension greater than 0 , maybe in terms of vanishing homology. This question is open even for the 0-degree Milnor class, i.e., the Milnor number, though for the top Milnor class at a non-singular component of $\operatorname{Sing}(V)$, Corollary 5.13 gives a positive answer.

We would like to thank Lê Dũng Tráng and Shoji Yokura for valuable conversations. While working on this article we visited various institutions and we are grateful, in particular, to Institut de Mathématiques de Luminy, Université de Montpellier, Instituto de Matemáticas UNAM at Cuernavaca and Hokkaido University, for their support and hospitality. We also acknowledge the financial support of CNRS, CONACYT and the Ministry of ESC, Japan, with gratitude.

After the preparation of the manuscript we learned that, in the hypersurface case, the formula for the Milnor number in [PP1] has been generalized in PP2] to a formula for the Milnor class, which was conjectured in $[\mathrm{Y}$. In particular, for a non-singular component $S$ of the singular set of a hypersurface $V$, such that $V$ satisfies the Whitney condition along $S$, our formula in Corollary 5.13 coincides with the one in PP2]. 


\section{Classical Chern and Schwartz-MacPherson classes}

We refer to St Sc1 Sc2 BS for basics on the material in this section. In sections 1 and 2, all homology and cohomology groups are with integral coefficients.

In order to fix the notation, we recall the definition of the Chern classes via obstruction theory $\mathrm{St}$. We restrict ourselves to the case of the tangent bundle $T M$ of an almost complex manifold $M$ of dimension $2 m$, the general case being entirely analogous. The bundle $T M$ can be naturally thought of as a complex vector bundle of rank $m$, and in the following, the linear independence is over the complex numbers $\mathbb{C}$.

Definition 1.1. An $r$-field on a subset $A$ in $M$ is an ordered set $F^{(r)}=\left\{v_{1}, \ldots, v_{r}\right\}$ of $r$ continuous vector fields on $A$. A singular point of $F^{(r)}$ is a point where the vectors $\left(v_{i}\right)$ fail to be linearly independent. An $r$-frame is a non-singular $r$-field.

The Stiefel manifold of $r$-frames in $\mathbb{C}^{m}$, denoted by $W_{r}(m)$, is $(2 m-2 r)$ connected and its first non-zero homotopy group is $\pi_{2 m-2 r+1}\left(W_{r}(m)\right) \simeq \mathbb{Z}$. The bundle of $r$-frames on $M$, denoted by $W_{r}(T M)$, is the bundle associated with the tangent bundle, whose fiber at $x \in M$ is the set of $r$-frames in $T_{x} M$, diffeomorphic to $W_{r}(m)$. In the following, we set $q=m-r+1$. The Chern class $c^{q}(M) \in H^{2 q}(M)$ is the primary obstruction to constructing a section of $W_{r}(T M)$. To define it, let $\sigma$ be a $k$-cell of a given (oriented) cellular decomposition $(D)$ of $M$, contained in an open subset $\Omega \subset M$ on which the bundle $W_{r}(T M)$ is trivialized. If the section $F^{(r)}$ of $W_{r}(T M)$ is already defined over the boundary of $\sigma$, it defines a map

$$
\left.\partial \sigma \simeq S^{k-1} \stackrel{F^{(r)}}{\longrightarrow} W_{r}(T M)\right|_{\Omega} \simeq \Omega \times W_{r}(m) \stackrel{p r_{2}}{\longrightarrow} W_{r}(m),
$$

thus an element of $\pi_{k-1}\left(W_{r}(m)\right)$. If $k \leq 2 m-2 r+1$, this homotopy group is zero, so the section $F^{(r)}$ can be extended inside $\sigma$ without singularity. If $k=2 q$, we meet an obstruction $I\left(F^{(r)}, \sigma\right) \in \pi_{2 q-1}\left(W_{r}(m)\right) \simeq \mathbb{Z}$. This defines a cochain,

$$
\gamma \in C^{2 q}\left(M ; \pi_{2 q-1}\left(W_{r}(m)\right)\right),
$$

by $\gamma(\sigma)=I\left(F^{(r)}, \sigma\right)$ for each $2 q$-cell $\sigma$, then extending it by linearity. This cochain is actually a cocycle and it represents the $q$-th Chern class $c^{q}(M)$ of $M$ in $H^{2 q}(M)$. It is independent of the various choices involved in its definition. Note that $c^{m}(M)$ coincides with the Euler class of the tangent bundle of $M$.

Hereafter, for a cellular decomposition $(D)$ of $M$, we denote by $D^{i}$ the $i$-skeleton of $(D)$. Let us denote by $L$ a $(D)$-subcomplex of $M$ and assume we are given an $r$-frame $F^{(r)}$ on $L \cap D^{2 q}$. The same arguments as before say that we can extend $F^{(r)}$ without singularity to $\left(L \cap D^{2 q}\right) \cup D^{2 q-1}$. This gives rise to a $2 q$-cochain which vanishes on $L \cap D^{2 q}$ and represents the relative Chern class

$$
c^{q}\left(M, L ; F^{(r)}\right) \in H^{2 q}(M, L),
$$

whose image by the natural map to $H^{2 q}(M)$ is the usual Chern class, however as a relative class it does depend on the choice of the frame $F^{(r)}$ on $L$.

If we have two frames $F_{1}^{(r)}$ and $F_{2}^{(r)}$ on $L \cap D^{2 q}$, the difference between the corresponding classes is given by the difference cocycle; in the product $L \times I$, suppose $F_{1}^{(r)}$ is defined at the level $L \times\{0\}$ and $F_{2}^{(r)}$ is defined at the level $L \times\{1\}$, then the difference cocycle $d\left(F_{1}^{(r)}, F_{2}^{(r)}\right)$ is well defined in

$$
H^{2 q}(L \times I, L \times\{0\} \cup L \times\{1\}) \simeq H^{2 q-1}(L)
$$


as the obstruction to the extension of the given sections on the boundary of $L \times I$ St, $§ 33.3$. As shown in St, we have

$$
c^{q}\left(M, L ; F_{2}^{(r)}\right)=c^{q}\left(M, L ; F_{1}^{(r)}\right)+\delta d\left(F_{1}^{(r)}, F_{2}^{(r)}\right),
$$

where $\delta: H^{2 q-1}(L) \longrightarrow H^{2 q}(M, L)$ is the connecting homomorphism.

We will use the relative Chern class in the case of a manifold with boundary $L=\partial M$ and we will denote

$$
c_{r-1}\left(M ; F^{(r)}\right)=c^{q}\left(M, \partial M ; F^{(r)}\right) \frown[M, \partial M] \in H_{2 r-2}(M) .
$$

We now consider a compact complex analytic variety $V$ of dimension $n$ embedded in a complex manifold $M$ of dimension $m$. We take a Whitney stratification $\left\{V_{i}\right\}$ of $M$ compatible with $V$ so that the singular part $\operatorname{Sing}(V)$ of $V$ is a union of strata (cf. [W]).

Let $(K)$ be a barycentric subdivision of a triangulation of $M$ compatible with the stratification $\left\{V_{i}\right\}$. We denote by $(D)$ the cellular decomposition of $M$ dual to $(K)$, which is defined by taking the first barycentric subdivision $\left(K^{\prime}\right)$ of $(K)$. We endow $(K)$ with an orientation compatible with the orientation of the stratification $\left\{V_{i}\right\}$. This determines an orientation for $(D)$ (see Br1]).

The union of (closed) cells which meet $V$ (or equivalently of cells dual to the $(K)$-simplices contained in $V$ ) is called a cellular tube around $V$, denoted by $\tilde{\mathcal{T}}_{V}$, and its boundary, the union of cells of $\tilde{\mathcal{T}}_{V}$ which are not dual to simplices in $V$, is denoted by $\partial \tilde{\mathcal{T}}_{V}$.

With the previous stratification and triangulation of $M$, we notice that the cells of $(D)$ are transverse to each stratum, so that, for every stratum $V_{i}$ of complex dimension $d$ and every cell $\sigma$ of (real) dimension $2 q=2(m-r+1), V_{i} \cap \sigma$ is a cell of dimension $2(d-r+1)$. In particular $V_{i} \cap \sigma$ is empty when $d<r-1$.

Let us recall that the obstruction dimension to construct an $r$-frame tangent to $M$ is $2 q=2(m-r+1)$, and the index $I\left(F^{(r)}, \sigma\right) \in \pi_{2 q-1}\left(W_{r}(m)\right)$ is well defined for every $2 q$-cell $\sigma$. We remark that the obstruction dimension to construct an $r$-frame tangent to the stratum $V_{i}$ of (complex) dimension $d$ is $2(d-r+1)=\operatorname{dim}\left(V_{i} \cap \sigma\right)$.

Definition 1.4. Let $A$ be a subspace of $M$. A stratified vector field on $A$ is a continuous section $v$ of $T M$ over $A$, such that at each point $x \in V_{i} \cap A, v(x)$ is tangent to the stratum $V_{i}$. A stratified $r$-field on $A$ is an $r$-field $F^{(r)}=\left\{v_{1}, \ldots, v_{r}\right\}$ consisting of stratified vector fields $v_{1}, \ldots, v_{r}$. A stratified $r$-frame is a non-singular stratified $r$-field.

The Schwartz classes are the primary obstruction to constructing special stratified frames on $V$ called radial frames. We list some of the main properties of a radial $r$-field $F_{0}^{(r)}$ on $\tilde{\mathcal{T}}_{V} \cap D^{2 q}$, denoting $F_{0}^{(r)}=\left(F_{0}^{(r-1)}, v_{0}\right)$ with $F_{0}^{(r-1)}$ the $(r-1)$-field of first $r-1$ vectors in $F_{0}^{(r)}$ and $v_{0}$ the last vector field:

(i) $F_{0}^{(r-1)}$ does not have singularities on $\tilde{\mathcal{T}}_{V} \cap D^{2 q} . F_{0}^{(r)}$ does not have singularities on $\tilde{\mathcal{T}}_{V} \cap D^{2 q-1}$ and has, for each $2 q$-cell $\sigma$, at most an isolated singularity at the barycenter of $\sigma$, which is the singularity of $v_{0}$.

(ii) If $F_{0}^{(r)}$ has a singularity in $\sigma$ and if $\sigma$ intersects with several strata, then the singularity is in the stratum $V_{i}$ of the lowest dimension. Let the dimension of $V_{i}$ be $d$. If $d>r-1$, then $I\left(F_{0}^{(r)}, \sigma\right)=I\left(\left.F_{0}^{(r)}\right|_{V_{i}}, V_{i} \cap \sigma\right)$, and if $d=r-1$, then $I\left(F_{0}^{(r)}, \sigma\right)=1$. 
(iii) The frame $F_{0}^{(r)}$ is everywhere pointing outwards from cellular tubes around $V$ and cellular tubes around the strata $V_{i}$ in a sense made precise in [BS] $\S \S 7$ and 8].

The motivation for considering radial frames is the following. For a radial vector field $v_{0}$ with isolated singularities $a_{i}$ in $V$, one has a Poincaré-Hopf theorem Sc2]

$$
\sum_{i} I\left(v_{0}, a_{i}\right)=\chi(V)
$$

independently of the ambient manifold $M$. This fundamental property of the Schwartz radial vector fields is not verified for arbitrary vector fields, using the classical definition of index.

The radial $r$-frame $F_{0}^{(r)}$ determines a $2 q$-cochain $\tilde{c}^{q} \in C^{2 q}\left(\tilde{\mathcal{T}}_{V}, \partial \tilde{\mathcal{T}}_{V}\right)$ on $M$ defined by $\tilde{c}^{q}(\sigma)=I\left(F_{0}^{(r)}, \sigma\right)$ if $\sigma$ is a $2 q$-cell intersecting $V$ and $\tilde{c}^{q}(\sigma)=0$ for the others, then extended by linearity. It is proved in $[\mathrm{Sc1}, \overline{\mathrm{BS}}$ that this cochain is actually a cocycle, representing a class $\tilde{c}^{q}(V) \in H^{2 q}\left(\tilde{\mathcal{T}}_{V}, \partial \tilde{\mathcal{T}}_{V}\right) \simeq H^{2 q}(M, M \backslash V)$. This class does not depend on the choices of the Whitney stratification of $M$, the triangulations, or the radial $r$-frame $F_{0}^{(r)}$ (see [Sc1], [Sc3]).

Definition 1.5. The $q$-th Schwartz class of $V$ is $\tilde{c}^{q}(V) \in H^{2 q}(M, M \backslash V)$.

Theorem 1.6 [BS]. For $r=1, \ldots, m$ and $q=m-r+1$, the image of the Schwartz class $\tilde{c}^{q}(V)$ by the Alexander duality isomorphism $H^{2 q}(M, M \backslash V) \stackrel{\sim}{\longrightarrow} H_{2 r-2}(V)$, is the corresponding MacPherson class of $V$ (defined in [Ma]),

$$
c_{r-1}(V) \in H_{2 r-2}(V) \text {. }
$$

Thus we call $c_{*}(V)=\sum_{r} c_{r}(V) \in H_{*}(V)$ the Schwartz-MacPherson (or simply $\mathrm{SM})$ class of $V$.

\section{Local Schwartz Class of a FRAme}

As in the previous section, the singular set of $V$ is denoted $\operatorname{Sing}(V)$ and the regular one $V_{0}=V \backslash \operatorname{Sing}(V)$. Let $S$ be either a compact connected $(K)$-subcomplex in $V_{0}$ or a connected component of $\operatorname{Sing}(V)$. Let $\tilde{U}$ be a neighborhood of $S$ in $M$, set $U=\tilde{U} \cap V$, and assume that $U \backslash S$ is in $V_{0}$.

Definition 2.1. A compact neighborhood $\tilde{\mathcal{T}}$ of $S$ in $M$, contained in $\tilde{U}$, such that $\tilde{\mathcal{T}} \backslash S$ retracts to $\partial \tilde{\mathcal{T}}$ and $\tilde{\mathcal{T}}$ retracts to $S$, will be called a tube in $\tilde{U}$ around $S$.

Definition 2.2. A cellular tube around $S$ in $M$, denoted by $\tilde{\mathcal{T}}$, is the union of (closed) cells of $(D)$ which are dual to simplices in $S$. The boundary $\partial \tilde{\mathcal{T}}$ is the union of cells in $\tilde{\mathcal{T}}$ which do not meet $S$.

Taking a subtriangulation, if necessary, we may assume that the cellular tube $\tilde{\mathcal{T}}$ is contained in $\tilde{U}$. On the other hand, $\partial \tilde{\mathcal{T}}$ is transverse to $V_{0}$. The intersection $\mathcal{T}=\tilde{\mathcal{T}} \cap V$ is a tube around $S$ in $U=\tilde{U} \cap V$ but is no more a cellular tube in $V$, if $S$ is a component of $\operatorname{Sing}(V)$. The boundary $\partial \mathcal{T}=V \cap \partial \tilde{\mathcal{T}}$ is a hypersurface in $V_{0}$.

There is a classical Alexander isomorphism, for $0 \leq q \leq m$,

$$
\psi_{M}: H^{2 q}(\tilde{U}, \tilde{U} \backslash S) \simeq H^{2 q}(\tilde{\mathcal{T}}, \partial \tilde{\mathcal{T}}) \stackrel{\sim}{\longrightarrow} H_{2 m-2 q}(S),
$$

when $S$ is a compact subcomplex of the complex manifold $M$ of dimension $m$. In the situation $S \subset V \subset M$ considered now, there is an Alexander homomorphism 
(in general not an isomorphism), for each $0 \leq p \leq n=\operatorname{dim}_{\mathbb{C}} V$,

$$
\psi_{V}: H^{2 p}(U, U \backslash S) \simeq H^{2 p}(\mathcal{T}, \partial \mathcal{T}) \longrightarrow H_{2 n-2 p}(S) .
$$

At the chain level, $\psi_{V}$ corresponds to the composition

$$
C_{\left(K^{\prime}\right)}^{2 p}(\mathcal{T}, \partial \mathcal{T}) \stackrel{\tau}{\longrightarrow} C_{(D)}^{2 q}(\tilde{\mathcal{T}}, \partial \tilde{\mathcal{T}}) \stackrel{\psi_{M}}{\longrightarrow} C_{2 m-2 q}^{(K)}(S)
$$

with $2 m-2 q=2 n-2 p$, and the map $\tau$ is defined by $\langle\tau(c), \sigma\rangle=\langle c, \sigma \cap V\rangle$ for a $2 q$-cell $\sigma$ in $(D)$ (see Br1] for details). The map $\tau$ induces homomorphisms, also denoted $\tau$ and called Thom-Gysin homomorphisms, making the diagram below commutative:

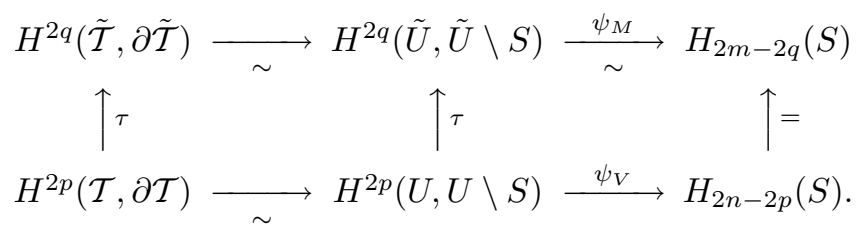

With the previous notation, we can suppose that $U \backslash S$ intersects $D^{2 q}$ in $V_{0}$. Let us write $r=m-q+1=n-p+1$

First suppose $S$ is in $V_{0}$. In this case $\psi_{V}$ is an isomorphism. For an $r$-frame $F^{(r)}$ on $(U \backslash S) \cap D^{2 q}$, we have the relative Chern class $c^{p}\left(\mathcal{T}, \partial \mathcal{T} ; F^{(r)}\right) \in H^{2 p}(\mathcal{T}, \partial \mathcal{T})$. The Poincaré-Hopf class of $F^{(r)}$ at $S$ is defined by

$$
\operatorname{PH}\left(F^{(r)}, S\right)=\psi_{V} c^{p}\left(\mathcal{T}, \partial \mathcal{T} ; F^{(r)}\right) \in H_{2 r-2}(S) .
$$

For a vector field $v$ and $S=\{a\}$ a point, $\operatorname{PH}(v, a)$ is the index previously denoted by $I(v, a)$.

Now we suppose $S$ may be a component of $\operatorname{Sing}(V)$. Let $F_{1}^{(r)}$ and $F_{2}^{(r)}$ be $r$ frames on $(U \backslash S) \cap D^{2 q}$, and let us consider a tube $\mathcal{T}$ in $U$ around $S$. The difference $d\left(F_{1}^{(r)}, F_{2}^{(r)}\right)$ is defined in $H^{2 p-1}(\partial \mathcal{T})$. Let $\delta: H^{2 p-1}(\partial \mathcal{T}) \longrightarrow H^{2 p}(\mathcal{T}, \partial \mathcal{T})$ be the connecting homomorphism, we set

$$
d_{S}\left(F_{1}^{(r)}, F_{2}^{(r)}\right)=\psi_{V} \delta d\left(F_{1}^{(r)}, F_{2}^{(r)}\right) \in H_{2 r-2}(S)
$$

Recall that, from the Schwartz construction, there exist radial $r$-fields on $U \cap D^{2 q}$ whose singularities are all located on $S$.

Definition 2.5. For an $r$-frame $F^{(r)}$ on $(U \backslash S) \cap D^{2 q}$, we define the Schwartz class $\operatorname{Sch}\left(F^{(r)}, S\right)$ of $F^{(r)}$ at $S$ to be the class in $H_{2 r-2}(S)$ given by

$$
\operatorname{Sch}\left(F^{(r)}, S\right)=c_{r-1}(S)+d_{S}\left(F_{0}^{(r)}, F^{(r)}\right)
$$

where $F_{0}^{(r)}$ is a radial frame.

If $S$ is in the non-singular part, $\operatorname{Sch}\left(F^{(r)}, S\right)$ coincides with $\mathrm{PH}\left(F^{(r)}, S\right)$. From the definition and (1.2), we get, for two $r$-frames $F_{1}^{(r)}$ and $F_{2}^{(r)}$ on $(U \backslash S) \cap D^{2 q}$,

$$
\operatorname{Sch}\left(F_{2}^{(r)}, S\right)=\operatorname{Sch}\left(F_{1}^{(r)}, S\right)+d_{S}\left(F_{1}^{(r)}, F_{2}^{(r)}\right)
$$

Let us consider now a neighborhood $U$ of $\operatorname{Sing}(V)$ in $V$. We know already that there exist stratified $r$-fields on $U \cap D^{2 q}$ whose singularities are all located in $\operatorname{Sing}(V)$. Elementary obstruction theory St tells us that every such $r$-field can be extended to $V_{0} \cap D^{2 q}$ with a singular set which is a subcomplex of $V_{0}$. More generally, let $\Sigma$ be a compact $(K)$-subcomplex in $V_{0}$ disjoint from a neighborhood $U_{1}$ of $\operatorname{Sing}(V)$ in $V$. We set $V^{*}=V \backslash U_{1}$ and let $\iota$ be the inclusion $V^{*} \hookrightarrow V$, 
which induces a homomorphism $\iota_{*}$ in homology with compact supports. Also, for a connected component $S$ of $\operatorname{Sing}(V) \cup \Sigma$, let $i$ be the inclusion $S \hookrightarrow V$. The following decomposition theorem follows from the previous discussion.

Theorem 2.7. Let $V$ be a compact analytic variety of dimension $n$ embedded in a complex manifold $M$ of dimension $m$ and let $\Sigma$ be a subset of $V_{0}$ as above. For any $r$-frame $F^{(r)}$ on $\left(V_{0} \backslash \Sigma\right) \cap D^{2 q}, q=m-r+1$, we have

$$
\sum_{S \subset \operatorname{Sing}(V)} i_{*} \operatorname{Sch}\left(F^{(r)}, S\right)+\iota_{*} c_{r-1}\left(V^{*} ; F^{(r)}\right)=c_{r-1}(V),
$$

where the sum is taken over the connected components of $\operatorname{Sing}(V), c_{r-1}(V)$ is the $S M$ class of $V$ and $c_{r-1}\left(V^{*} ; F^{(r)}\right)$ is the Chern class of $V^{*}$ relative to $F^{(r)}$ (cf. 1.3), so that

$$
\iota_{*} c_{r-1}\left(V^{*} ; F^{(r)}\right)=\sum_{S \subset \Sigma} i_{*} \operatorname{PH}\left(F^{(r)}, S\right) .
$$

In other words, this result says that an appropriate $r$-frame gives a splitting of the corresponding SM class in two parts; one localized in the singular set of $V$, the other being the usual Chern class of the regular part relative to the $r$-frame.

\section{Differential Geometric Viewpoint}

In this section, and from now on, all homology and cohomology groups will be with real coefficients. The (co)homology classes previously defined with integral coefficients will be looked in this context. The general settings, in particular concerning the Chern-Weil theory and the integration on the Cech-de Rham cohomology, can be found in [Bo], [BB], Le1], Le2], [Su2].

In general, for a Chern polynomial $\varphi$, i.e., a polynomial on the Chern classes, and a connection $\nabla$ for a complex $C^{\infty}$ vector bundle $E$, we denote by $\varphi(\nabla)$ the cocycle on the base space which is the image of $\varphi$ by the Chern-Weil homomorphism associated with $\nabla$. It is a closed form whose class is the characteristic class $\varphi(E)$ of the bundle $E$ with respect to $\varphi$. In particular, the class of $c^{i}(\nabla)$ is the $i$-th Chern class $c^{i}(E)$. If $\left(\nabla_{0}, \ldots, \nabla_{r}\right)$ is a family of $r+1$ connections for $E, \varphi\left(\nabla_{0}, \ldots, \nabla_{r}\right)$ will denote the Bott difference operator [Bo], so that

$$
d \varphi\left(\nabla_{0}, \ldots, \nabla_{r}\right)=\sum_{i=0}^{r}(-1)^{i} \varphi\left(\nabla_{0}, \ldots, \widehat{\nabla}_{i}, \cdots, \nabla_{r}\right) .
$$

Now let $S \subset V \subset M$ be as in section 2. Let $\tilde{U}$ be a neighborhood of $S$ in $M$ such that $U \backslash S$ is in $V_{0}$, with $U=\tilde{U} \cap V$. As in section 2, we consider a $(D)$-cellular tube $\tilde{\mathcal{T}}$ around $S$ in $\tilde{U}$. Let us denote by $\left(D^{\prime}\right)$ the cellular decomposition of $M$ dual to $\left(K^{\prime}\right)$. The cells of $\left(D^{\prime}\right)$ consist of simplices of the second barycentric subdivision $\left(K^{\prime \prime}\right)$ of $(K)$. We denote by $\tilde{\mathcal{R}}$ the $\left(D^{\prime}\right)$-cellular tube around $S$. Thus $\tilde{\mathcal{R}}$ is in the interior of $\tilde{\mathcal{T}}$ and the $(D)$-cells are transverse to $\partial \tilde{\mathcal{R}}$. We endow $\partial \tilde{R}$ with the ordinary orientation as the boundary. We set $\mathcal{R}=\tilde{\mathcal{R}} \cap V$.

Suppose we have an $r$-frame $F^{(r)}$ on $(U \backslash S) \cap D^{2 q}, q=m-r+1$, we may describe the Schwartz class $\operatorname{Sch}\left(F^{(r)}, S\right)$ of $F^{(r)}$ at $S$ as follows.

First we consider the case where $S$ is in the regular part $V_{0}$ of $V$ (thus $U$ is also in $\left.V_{0}\right)$ and give a differential geometric interpretation of the Poincaré-Hopf class $\operatorname{PH}\left(F^{(r)}, S\right)$. The relative class $c^{p}\left(\mathcal{T}, \partial \mathcal{T} ; F^{(r)}\right)$ in section 2 is now defined by taking an " $F^{(r)}$-trivial connection" for $T U$ away from $S$. To be more precise, let 

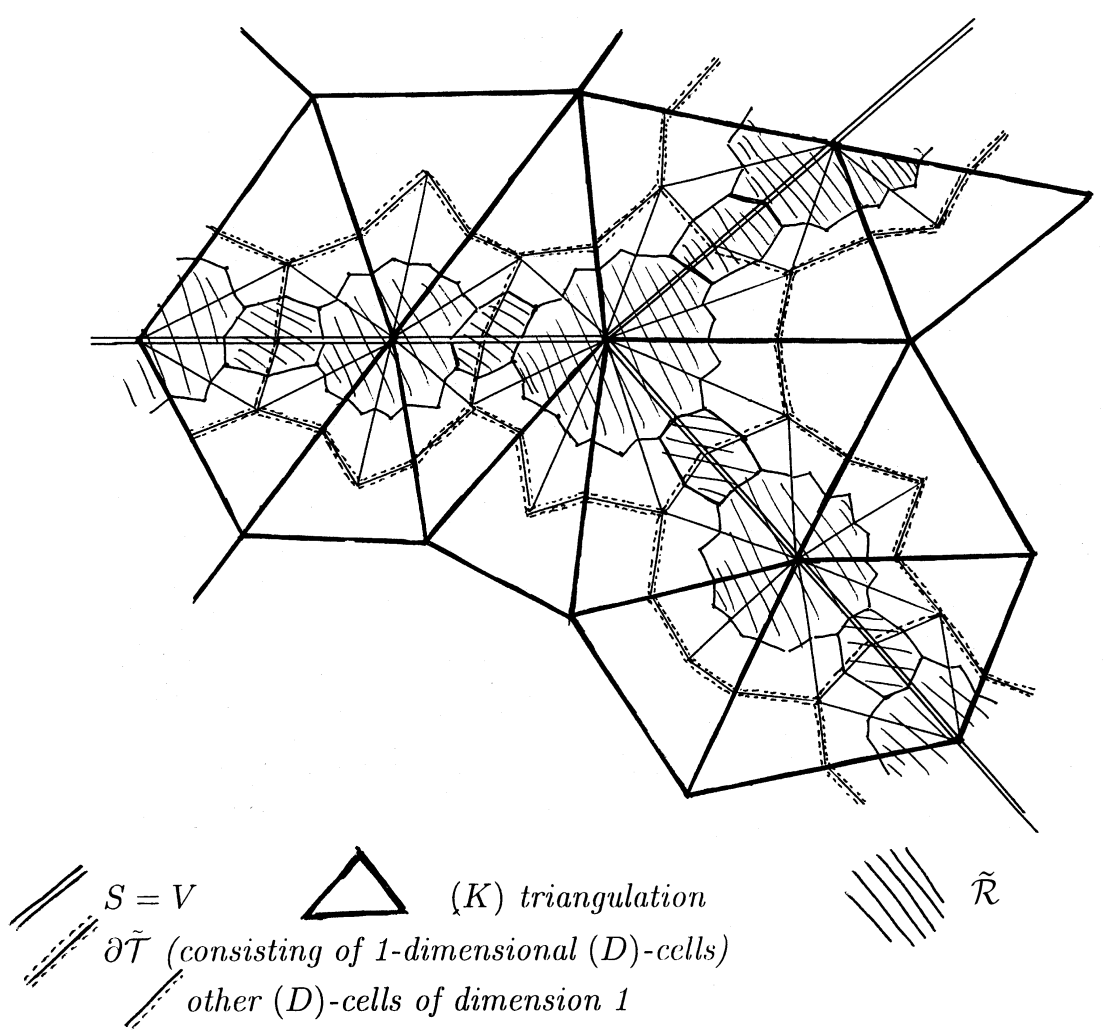

other $(D)$-cells of dimension 1

For such a $(D)$-cell $\sigma$ of dimension 1 intersecting $V$, the boundary $\partial \widetilde{\mathcal{R}}$ is transverse to $\sigma$ in their intersecting points.

FIGURE 1.

$\nabla$ be a connection for $T U$ on $U$ and let $\nabla_{0}$ be an $F^{(r)}$-trivial connection for $T U$ on a neighborhood of $(U \backslash S) \cap D^{2 q}$ in $U$. Here $\nabla_{0}$ being $F^{(r)}$-trivial means that $\nabla_{0}(v)=0$ for every member $v$ of $F^{(r)}$ so that $c^{p}\left(\nabla_{0}\right)=0$ (see, for example, Su2, Ch.II, 9). Then the image $\xi$ of $c^{p}\left(\mathcal{T}, \partial \mathcal{T} ; F^{(r)}\right)$ by the Thom-Gysin homomorphism $\tau: H^{2 p}(U, U \backslash S) \rightarrow H^{2 q}(\tilde{U}, \tilde{U} \backslash S)$ is represented by the cocycle

$$
\gamma \mapsto \int_{\gamma \cap \mathcal{R}} c^{p}(\nabla)+\int_{\gamma \cap \partial \mathcal{R}} c^{p}\left(\nabla, \nabla_{0}\right),
$$

for a relative cycle $\gamma \in C_{2 q}^{(D)}(\tilde{\mathcal{T}}, \partial \tilde{\mathcal{T}})$ [Le1]-[Le2], where $C_{k}^{(D)}(A)$ denotes the chains of dimension $k$ in the $(D)$-complex $A$. The Poincaré-Hopf class $\mathrm{PH}\left(F^{(r)}, S\right)$ is then given by $\psi_{V} c^{p}\left(\mathcal{T}, \partial \mathcal{T} ; F^{(r)}\right)=\psi_{M} \xi($ see $(2.3))$.

Now suppose $S$ may be a component of $\operatorname{Sing}(V)$ and let $F_{0}^{(r)}$ be a radial $r$ frame on $(\tilde{U} \backslash S) \cap D^{2 q}$. Recall that the Schwartz class of $F_{0}^{(r)}$ at $S$ is given by $\operatorname{Sch}\left(F_{0}^{(r)}, S\right)=c_{r-1}(S)=\psi_{M} \tilde{c}^{q}(S)$, where $\tilde{c}^{q}(S) \in H^{2 q}(\tilde{\mathcal{T}}, \partial \tilde{\mathcal{T}}) \simeq H^{2 q}(\tilde{U}, \tilde{U} \backslash S)$ is the $q$-th Schwartz class of $S$. We may assume that $F_{0}^{(r)}$ is given on a neighborhood $\tilde{W}$ of $(\tilde{U} \backslash S) \cap D^{2 q}$. We denote by $\tilde{\nabla}$ a connection for $T M$ on $\tilde{U}$ and by $\tilde{\nabla}_{0}$ an $F_{0}^{(r)}$-trivial connection for $T M$ on $\tilde{W}$. From the definitions, we have the following. 
Proposition 3.3. The relative class $\tilde{c}^{q}(S)$ is represented by the cocycle

$$
\gamma \mapsto \int_{\gamma \cap \tilde{\mathcal{R}}} c^{q}(\tilde{\nabla})+\int_{\gamma \cap \partial \tilde{\mathcal{R}}} c^{q}\left(\tilde{\nabla}, \tilde{\nabla}_{0}\right)
$$

for a relative cycle $\gamma \in C_{2 q}^{(D)}(\tilde{\mathcal{T}}, \partial \tilde{\mathcal{T}})$.

A differential geometric description for the Schwartz class of a general frame is obtained by combining the above and the following formula for the difference cocycle, introduced earlier (see 2.4). Let $S$ be either a compact connected $(K)$ subcomplex in $V_{0}$ or a connected component of $\operatorname{Sing}(V)$ as before. Let $F_{1}^{(r)}$ and $F_{2}^{(r)}$ be two $r$-frames on $(U \backslash S) \cap D^{2 q}$. We may assume that $F_{1}^{(r)}$ and $F_{2}^{(r)}$ are given on a neighborhood $W$ of $(U \backslash S) \cap D^{2 q}$ in $U$. For each $i=1,2$, let $\nabla_{i}$ be an $F_{i}^{(r)}$-trivial connection for $T V_{0}$ on $W$.

Lemma 3.4. The difference $\delta d\left(F_{1}^{(r)}, F_{2}^{(r)}\right)$ is an element in $H^{2 p}(U, U \backslash S)$ whose image by the Thom Gysin homomorphism $\tau: H^{2 p}(U, U \backslash S) \rightarrow H^{2 q}(\tilde{U}, \tilde{U} \backslash S)$ is represented by the cocycle

$$
\gamma \mapsto \int_{\gamma \cap \partial \mathcal{R}} c^{p}\left(\nabla_{1}, \nabla_{2}\right)
$$

for a relative cycle $\gamma \in C_{2 q}^{(D)}(\tilde{\mathcal{T}}, \partial \tilde{\mathcal{T}})$.

Proof. Note that the lemma directly follows from (3.2) if $S$ is in $V_{0}$. Now suppose that $S$ may be a connected component of $\operatorname{Sing}(V)$. Let $\mathcal{R}_{1}$ be another $\left(D^{\prime}\right)$-tube around $S$ contained in the interior of $\mathcal{R}$ and set $C=\mathcal{R} \backslash \mathcal{R}_{1}$. Then $\partial C=\partial \mathcal{R}-\partial \mathcal{R}_{1}$. Let $\tilde{\nabla}$ be a connection for $T V_{0}$ on $C \cap W$ which extends simultaneously $\nabla_{1}$ on $\partial \mathcal{R}_{1} \cap W$ and $\nabla_{2}$ on $\partial \mathcal{R} \cap W$. Let $\tilde{\nabla}_{1}$ be an $F_{1}^{(r)}$-trivial connection for $T V_{0}$ on $C \cap W$ which extends $\nabla_{1}$ on $\partial \mathcal{R}_{1} \cap W$. Thus we have $c^{p}\left(\tilde{\nabla}_{1}\right)=0$, hence $d c^{p}\left(\tilde{\nabla}_{1}, \tilde{\nabla}\right)=c^{p}(\tilde{\nabla})$. Let $F^{(r)}$ be an $r$-frame which coincides with $F_{1}^{(r)}$ near $\partial \mathcal{R}_{1}$ and with $F_{2}^{(r)}$ near $\partial \mathcal{R}$. Note that $C$ is homotopically equivalent to $\partial \mathcal{R} \times I$ and that $d\left(F_{1}^{(r)}, F_{2}^{(r)}\right)=c^{p}\left(C, \partial C ; F^{(r)}\right) \in H^{2 p}(C, \partial C) \simeq H^{2 p-1}(\partial \mathcal{R})$. The image of the class $\delta d\left(F_{1}^{(r)}, F_{2}^{(r)}\right)$ by $\tau$ is represented by the cocycle $\gamma \rightarrow \int_{\gamma \cap C} c^{p}(\tilde{\nabla})$ for a relative $2 q$-cycle $\gamma$. By the Stokes formula $\int_{\gamma \cap C} c^{p}(\tilde{\nabla})=\int_{\gamma \cap \partial C} c^{p}\left(\tilde{\nabla}_{1}, \tilde{\nabla}\right)=$ $\int_{\gamma \cap \partial \mathcal{R}} c^{p}\left(\nabla_{1}, \nabla_{2}\right)$.

\section{Virtual Class}

We now suppose that $V$ is a compact local complete intersection of dimension $n$ in a manifold $M$ of dimension $m$, defined as the zero set of a holomorphic section $s$ of a holomorphic vector bundle $N$ of rank $k=m-n$ over $M$. The restriction $\left.N\right|_{V_{0}}$ coincides with the normal bundle $N_{V_{0}}$ of $V_{0}=V \backslash \operatorname{Sing}(V)$ in $M$ and we have an exact sequence of vector bundles,

$$
\left.0 \rightarrow T V_{0} \rightarrow T M\right|_{V_{0}} \stackrel{\pi}{\rightarrow} N_{V_{0}} \rightarrow 0 .
$$

We call $\tau_{V}=\left.(T M-N)\right|_{V}$ the virtual tangent bundle of $V$. Let us remark that under the previous assumptions, the virtual tangent bundle does not depend on the choice of $(N, s)$. 
The total Chern class $c^{*}(T M-N) \in H^{*}(M)$ of the virtual bundle $T M-N$ is given by $c^{*}(T M-N)=c^{*}(T M) \cdot c^{*}(N)^{-1}$. Hence the $p$-th Chern $\operatorname{class} c^{p}(T M-N)$ is the coefficient of $t^{p}$ in the expansion of

$$
\left(1+\sum_{i=1}^{m} t^{i} c^{i}(T M)\right)\left(1+\sum_{j=1}^{k} t^{j} c^{j}(N)\right)^{-1}
$$

This polynomial may be written as a finite sum

$$
c^{p}(T M-N)=\sum_{\ell} \varphi_{\ell}^{(p)}\left(c^{1}(T M), \ldots, c^{m}(T M)\right) \cdot \psi_{\ell}^{(p)}\left(c^{1}(N), \ldots, c^{k}(N)\right),
$$

for suitable polynomials $\varphi_{\ell}^{(p)}$ and $\psi_{\ell}^{(p)}$. Let $\nabla$ and $\nabla^{\prime}$ be connections for $T M$ and $N$ respectively, defined on some submanifold $\Omega$ of $M$. Denoting by $\nabla^{\bullet}$ the pair $\left(\nabla, \nabla^{\prime}\right)$, we set

$$
c^{p}\left(\nabla^{\bullet}\right)=\sum_{\ell} \varphi_{\ell}^{(p)}(\nabla) \cdot \psi_{\ell}^{(p)}\left(\nabla^{\prime}\right)
$$

where the product is the exterior product. Then $c^{p}\left(\nabla^{\bullet}\right)$ is a closed $2 p$-form and defines the class $c^{p}(T M-N)$ on $\Omega$.

If $\left(\nabla_{0}^{\bullet}, \ldots, \nabla_{r}^{\bullet}\right)$ is a family of $r+1$ pairs of connections, $\nabla_{j}^{\bullet}=\left(\nabla_{j}, \nabla_{j}^{\prime}\right)$ with $\nabla_{j}$ connections for $T M$ and $\nabla_{j}^{\prime}$ for $N$, we may construct $c^{p}\left(\nabla_{0}^{\bullet}, \ldots, \nabla_{r}^{\bullet}\right)$ satisfying an identity similar to (3.1), as in Bo. Namely, let $\Delta^{r}$ be the standard simplex $\sum_{i=0}^{r} t_{j} \leq 1, t_{j} \geq 0$ in $\mathbb{R}^{r+1}$, and $\varpi: \Omega \times \Delta^{r} \rightarrow \Omega$ the natural projection. We set $\tilde{\nabla}^{\bullet}=\left(\tilde{\nabla}, \tilde{\nabla}^{\prime}\right)$, where $\tilde{\nabla}$ and $\tilde{\nabla}^{\prime}$ denote the connections for $\varpi^{*}(T M)$ and $\varpi^{*}(N)$ given by $\tilde{\nabla}=\left(1-\sum_{j=0}^{r} t_{j}\right) \nabla_{0}+\sum_{j=0}^{r} t_{j} \nabla_{j}$ and similarly for $\tilde{\nabla}^{\prime}$. Then, $c^{p}\left(\nabla_{0}^{\bullet}, \ldots, \nabla_{r}^{\bullet}\right)$ is equal to the integration of $c^{p}\left(\tilde{\nabla}^{\bullet}\right)$ along the fiber $\Delta^{r}$ of the projection $\varpi$.

Let $\Omega_{0}$ be a subset in $V_{0} \cap \Omega$. The pair $\nabla^{\bullet}=\left(\nabla, \nabla^{\prime}\right)$ will be said to be compatible on $\Omega_{0}$ if, on $\Omega_{0}$, the connection $\nabla^{\prime}$ is obtained from $\nabla$ by passing to the quotient, i.e., $\pi \circ \nabla=\nabla^{\prime} \circ \pi$. This implies that $\nabla$ induces a connection for $T V_{0}$, denoted by $\nabla^{V}$, and the triple $\left(\nabla^{V}, \nabla, \nabla^{\prime}\right)$ is compatible with $(4.1)$ in the sense of $[\mathrm{BB}], 4.16$.

Lemma 4.2. (i) If $\nabla^{\bullet}$ is a compatible pair on $\Omega_{0}$, then $c^{p}\left(\nabla^{\bullet}\right)=c^{p}\left(\nabla^{V}\right)$ on $\Omega_{0}$.

(ii) If $\nabla_{1}^{\bullet}$ and $\nabla_{2}^{\bullet}$ are two compatible pairs on $\Omega_{0}$, then $c^{p}\left(\nabla_{1}^{\bullet}, \nabla_{2}^{\bullet}\right)=c^{p}\left(\nabla_{1}^{V}, \nabla_{2}^{V}\right)$ on $\Omega_{0}$.

Proof. (i) is proved as in $[\mathrm{BB}], 4.22$. To prove (ii), let $\varpi: \Omega_{0} \times[0,1] \rightarrow \Omega_{0}$ be the projection and let $\tilde{\nabla}$ and $\tilde{\nabla}^{\prime}$ be connections for $\varpi^{*} T M$ and $\varpi^{*} N$, respectively, given by $\tilde{\nabla}=t \nabla_{2}+(1-t) \nabla_{1}$ and $\tilde{\nabla}^{\prime}=t \nabla_{2}^{\prime}+(1-t) \nabla_{1}^{\prime}$. Then the pair $\tilde{\nabla} \bullet=\left(\tilde{\nabla}, \tilde{\nabla}{ }^{\prime}\right)$ is compatible on $\Omega_{0} \times[0,1]$. Therefore, for the connection $\tilde{\nabla}^{V}=t \nabla_{2}^{V}+(1-t) \nabla_{1}^{V}$, we have $c^{p}\left(\tilde{\nabla}^{V}\right)=c^{p}\left(\tilde{\nabla}^{\bullet}\right)=\sum_{\ell} \varphi_{\ell}^{(p)}(\tilde{\nabla}) \cdot \psi_{\ell}^{(p)}\left(\tilde{\nabla}^{\prime}\right)$. The formula of (ii) is obtained by integration on $[0,1]$.

Letting $r=n-p+1$, the image of $c^{p}\left(\tau_{V}\right)$ by the Poincaré homomorphism $H^{2 p}(V) \rightarrow H_{2 r-2}(V)$ coincides with the FJ class [FJ], [F]:

$$
c_{r-1}^{F J}(V)=c^{p}\left(\tau_{V}\right) \frown[V]
$$

The virtual class of an $r$-frame is defined by localizing $c^{p}\left(\tau_{V}\right)$ by the frame.

To be more precise, let us consider a subset $S$ and suitable neighborhoods as in the previous sections. Let $F^{(r)}$ be an $r$-frame on $(U \backslash S) \cap D^{2 q}$. Let $\nabla$ and $\nabla^{\prime}$ be connections for $T M$ and $N$, respectively, on $\tilde{U}$ and set $\nabla^{\bullet}=\left(\nabla, \nabla^{\prime}\right)$. Also, 
let $\nabla_{0}$ and $\nabla_{0}^{\prime}$ be connections for $T M$ and $N$, respectively, on a neighborhood $W$ of $(U \backslash S) \cap D^{2 q}$ in $U$ such that the pair $\nabla_{0}^{\bullet}=\left(\nabla_{0}, \nabla_{0}^{\prime}\right)$ is compatible and $\nabla_{0}$ is $F^{(r)}$-trivial. If we consider the $2 q$-cochain given by

$$
\gamma \mapsto \int_{\gamma \cap \mathcal{R}} c^{p}\left(\nabla^{\bullet}\right)+\int_{\gamma \cap \partial \mathcal{R}} c^{p}\left(\nabla^{\bullet}, \nabla_{0}^{\bullet}\right), \quad \gamma \in C_{2 q}^{(D)}(\tilde{\mathcal{T}}, \partial \tilde{\mathcal{T}}),
$$

it is a cocycle independent of the choices of connections, with $\nabla_{0} F^{(r)}$-trivial, and defines an element $\eta$ in $H^{2 q}(\tilde{U}, \tilde{U} \backslash S)$.

Definition 4.4. We define the virtual class $\operatorname{Vir}\left(F^{(r)}, S\right)$ of $F^{(r)}$ at $S$ to be the image of $\eta$ by the Alexander isomorphism $\psi_{M}: H^{2 q}(\tilde{U}, \tilde{U} \backslash S) \rightarrow H_{2 r-2}(S)$.

Recall that, if $S$ is in $V_{0}$, the Poincaré-Hopf class $\mathrm{PH}\left(F^{(r)}, S\right) \in H_{2 r-2}(S)$ is dual to the class represented by the cocycle (3.2). Thus in this case, from Lemma 4.2 , we have

$$
\operatorname{Vir}\left(F^{(r)}, S\right)=\operatorname{PH}\left(F^{(r)}, S\right)
$$

The following formula for two $r$-frames $F_{1}^{(r)}$ and $F_{2}^{(r)}$ as above, analogous to (2.6), is a consequence of Lemmas 3.4 and 4.2.

$$
\operatorname{Vir}\left(F_{2}^{(r)}, S\right)=\operatorname{Vir}\left(F_{1}^{(r)}, S\right)+d_{S}\left(F_{1}^{(r)}, F_{2}^{(r)}\right)
$$

Also the following theorem, analogous to Theorem 2.7, follows from the previous discussion.

Theorem 4.6. Let $\Sigma$ be a subset in $V_{0}$ as in Theorem 2.7. With the above hypotheses and notation, if $F^{(r)}$ is an $r$-frame on $\left(V_{0} \backslash \Sigma\right) \cap D^{2 p}$, we have, in $H_{2 r-2}(V)$,

$$
\sum_{S \subset \operatorname{Sing}(V)} i_{*} \operatorname{Vir}\left(F^{(r)}, S\right)+\iota_{*} c_{r-1}\left(V^{*}, F^{(r)}\right)=c_{r-1}^{F J}(V),
$$

where the sum is taken over the connected components of the singular set $\operatorname{Sing}(V)$ and $c_{r-1}\left(V^{*} ; F^{(r)}\right)$ is the Chern class of $V^{*}$ relative to $F^{(r)}$, so that

$$
\iota_{*} c_{r-1}\left(V^{*} ; F^{(r)}\right)=\sum_{S \subset \Sigma} i_{*} \mathrm{PH}\left(F^{(r)}, S\right) .
$$

\section{Milnor Class}

Let $V$ be a local complete intersection of dimension $n$ defined by a section of a vector bundle $N$ over the ambient complex manifold $M$ of dimension $m$, as in the previous section. We introduce the Milnor classes of $V$ at a connected component $S$ of $\operatorname{Sing}(V)$. For $r \geq 1$, let $F^{(r)}$ be an $r$-frame on $(U \backslash S) \cap D^{2 q}$, where $U$ is a neighborhood of $S$ in $V$ such that $U \backslash S \subset V_{0}$ and $q=m-r+1$.

Definition 5.1. The $(r-1)$-st Milnor class $\mu_{r-1}(V, S)$ of $V$ at $S$ is defined by

$$
\mu_{r-1}(V, S)=(-1)^{n+1}\left(\operatorname{Sch}\left(F^{(r)}, S\right)-\operatorname{Vir}\left(F^{(r)}, S\right)\right) \quad \text { in } \quad H_{2 r-2}(S),
$$

which is independent of the choice of $F^{(r)}$ by (2.6) and (4.5).

We call $\mu_{*}(V, S)=\sum_{r \geq 0} \mu_{r}(V, S) \in H_{*}(S)$ the total Milnor class of $V$ at $S$. Note that $\mu_{r}(V, S)=0$ for $r>\operatorname{dim}_{\mathbb{C}} S$. Since there always exist frames as in Theorems 2.7 and 4.6 , we have 
Theorem 5.2. For a subvariety $V$ of a complex manifold $M$ as above,

$$
c_{*}(V)=c_{*}^{F J}(V)+(-1)^{n+1} \sum_{S} i_{*} \mu_{*}(V, S) \quad \text { in } \quad H_{*}(V),
$$

where the sum is taken over the connected components $S$ of $\operatorname{Sing}(V)$.

In particular, if the singularities of $V$ are isolated points, then the Milnor classes are zero, except in degree 0 where they coincide with the usual Milnor numbers of [Mi], [H], [Lo (see section 6). Hence, in this case the SM classes and the FJ classes of $V$ coincide in all dimensions, except in degree 0 , where their difference is given by the sum of the usual Milnor numbers, recovering the formula in [SS2], Su1].

Remarks 5.3. 1. The classes $\operatorname{PH}\left(F^{(r)}, S\right), \operatorname{Sch}\left(F^{(r)}, S\right)$ and $\operatorname{Vir}\left(F^{(r)}, S\right)$ may be defined for an $r$-frame $F^{(r)}$ on the intersection of a neighborhood of $\partial \mathcal{T}$ (in $V$ ) and $D^{2 q}$, where $\mathcal{T}=\tilde{\mathcal{T}} \cap V$ with $\tilde{\mathcal{T}}$ a cellular tube around $S$.

2. When $r=1$, i.e., $F^{(1)}=(v), \operatorname{PH}(v, S), \operatorname{Sch}(v, S)$ and $\operatorname{Vir}(v, S)$ are called, respectively, the Poincaré-Hopf, Schwartz and virtual indices of the vector field $v$ [GSV], LSS], SS1]-SS2]. The corresponding Milnor class $\mu_{0}(V, S)$ is a number which will be discussed in section 6 .

In the rest of this section, we prove a Lefschetz type formula for the Milnor classes at a non-singular connected component $S$ of the singular set of $V$ under the assumption that $V$ satisfies the Whitney condition along $S$.

Let $\tilde{U}$ be a tubular neighborhood of $S$ in $M$ with $C^{\infty}$ projection $\tilde{\rho}: \tilde{U} \rightarrow S$. We set $U=\tilde{U} \cap V$ and $U_{0}=U \backslash S$ and denote by $\rho$ and $\rho_{0}$, respectively, the restrictions of $\tilde{\rho}$ to $U$ and $U_{0}$. From the Whitney condition, we see that the fibers of $\tilde{\rho}$ are transverse to $V$ and that $S$ is a deformation retract of $U$ with retraction $\rho$. We identify $\rho_{0}^{*}\left(\left.N\right|_{S}\right)$ with $N_{U_{0}}$, and $\tilde{\rho}^{*}\left(\left.N\right|_{S}\right)$ with $\left.N\right|_{\tilde{U}}$. The bundle $T \tilde{\rho}$ of vectors in $T \tilde{U}$ tangent to the fibers of $\tilde{\rho}$ admits a complex structure, since it is $C^{\infty}$ isomorphic with the normal bundle of the complex submanifold $S$ in $W$. Let $\tilde{\mathcal{T}}$ be a $(D)$ cellular tube around $S$ in $\tilde{U}$ and $\tilde{\mathcal{R}}$ a $\left(D^{\prime}\right)$-cellular tube in $\tilde{\mathcal{T}}$ as in section 3 . We set $\mathcal{T}=\tilde{\mathcal{T}} \cap V$ and $\mathcal{R}=\tilde{\mathcal{R}} \cap V$ as before.

Let $s$ denote the complex dimension of $S$ and let $F^{(r-1)}$ be an $(r-1)$-frame on the $2(s-r+1)$-skeleton $S \cap D^{2 q}$ of $S$. In what follows, we set $\ell=s-r+1$. By the Schwartz construction, there exists a radial $r$-field $F_{0}^{(r)}=\left(F_{0}^{(r-1)}, v_{0}\right)$ on $\tilde{\mathcal{T}} \cap D^{2 q}$ such that $F_{0}^{(r-1)}$ extends $F^{(r-1)}$. The radial vector field $v_{0}$ is tangent to $U_{0}$ and possibly has singularities in the barycenters of $2 \ell$-cells in $S \cap D^{2 q}$. We may assume that $v_{0}$ is tangent to the fibers of $\tilde{\rho}$ near $\partial \tilde{\mathcal{R}}$.

Let $v$ be a vector field on $U_{0} \cap D^{2 q}$ which is non-singular and tangent to the fibers of $\rho$ in a neighborhood $U_{0}^{\prime}$ of $\partial \mathcal{R}$ so that $F^{(r)}=\left(F_{0}^{(r-1)}, v\right)$ is an $r$-frame on $U_{0}^{\prime} \cap D^{2 q}$. For example, the above $v_{0}$ has these properties.

For a point $x$ in $S \cap D^{2 q}$, let $\tilde{U}_{x}$ denote the fiber of $\tilde{\rho}$ at $x$ and set $U_{x}=\tilde{U}_{x} \cap V$, which is the fiber of $\rho$ at $x$. We also set $\mathcal{R}_{x}=\mathcal{R} \cap U_{x}$. The restriction of $v$ to $U_{x}$ determines the $\operatorname{Schwartz}$ index $\operatorname{Sch}(v, x)$ and the virtual index $\operatorname{Vir}(v, x)$ on $U_{x}$. By the Whitney condition, these indices do not depend on $x$.

Recall that we have the difference $d_{S}\left(F_{0}^{(r)}, F^{(r)}\right)$ in $H_{2 r-2}(S)$. We also have the difference $d\left(v_{0}, v\right)$, which is an integer, of $v_{0}$ and $v$ as vector fields on $U_{x}$.

Lemma 5.4. We have

$$
d_{S}\left(F_{0}^{(r)}, F^{(r)}\right)=d\left(v_{0}, v\right) \cdot c_{r-1}(S) .
$$


Proof. We consider the exact sequence of vector bundles on $U_{0}$ :

$$
0 \rightarrow T \rho_{0} \rightarrow T U_{0} \rightarrow \rho_{0}^{*} T S \rightarrow 0,
$$

where $T \rho_{0}$ denotes the bundle of vectors in $T U_{0}$ tangent to the fibers of $\rho_{0}$. We may assume that $F_{0}^{(r)}$ and $F^{(r)}$ are $r$-frames on a neighborhood $W$ of $U_{0}^{\prime} \cap D^{2 q}$. Let $\nabla_{1}^{\rho}$ and $\nabla_{2}^{\rho}$ be, respectively, $v_{0}$-trivial and $v$-trivial connections for $T \rho_{0}$ on $W$. Also let $\nabla^{S}$ be an $F_{0}^{(r-1)}$-trivial connection for $T S$ on a neighborhood of $S \cap D^{2 q}$. We take connections $\nabla_{1}$ and $\nabla_{2}$ for $T U_{0}$ so that $\left(\nabla_{1}^{\rho}, \nabla_{1}, \rho_{0}^{*} \nabla^{S}\right)$ and $\left(\nabla_{2}^{\rho}, \nabla_{2}, \rho_{0}^{*} \nabla^{S}\right)$ are both compatible with the above sequence. Thus $\nabla_{1}$ is $F_{0}^{(r)}$-trivial and $\nabla_{2}$ is $F^{(r)}$-trivial on $W$. By Lemma 3.4, the homology class $d_{S}\left(F_{0}^{(r)}, F^{(r)}\right)$ is determined by

$$
c^{p}\left(\nabla_{1}, \nabla_{2}\right)=\sum_{i+j=p} c^{i}\left(\nabla_{1}^{\rho}, \nabla_{2}^{\rho}\right) \cdot \rho_{0}^{*} c^{j}\left(\nabla^{S}\right)
$$

We recall the commutative diagram

$$
\begin{array}{ccc}
H^{2 q}(\tilde{U}, \tilde{U} \backslash S) & \stackrel{\sim}{\tilde{\rho}_{*}} H^{2 \ell}(S) \\
\imath \downarrow \psi_{M} & & \imath \downarrow \\
H_{2 r-2}(S) & \stackrel{=}{\longrightarrow} H_{2 r-2}(S),
\end{array}
$$

where the first row is the inverse of the Thom isomorphism, given by integration along the fibers of $\tilde{\rho}$, and the second column is Poincaré duality. The dual of the first row in (5.6) gives an isomorphism

$$
H_{2 q}(\tilde{U}, \tilde{U} \backslash S) \stackrel{\sim}{\longleftarrow} H_{2 \ell}(S),
$$

which shows that every relative $2 q$-cycle $\gamma$ (is homologous to a cycle which) fibers over a $2 \ell$-cycle $\zeta$ of $S$. By the projection formula, we get from (5.5) (note that the rank of the bundle $T \rho_{0}$ is $n-s$ )

$$
\int_{\gamma \cap \partial \mathcal{R}} c^{p}\left(\nabla_{1}, \nabla_{2}\right)=\int_{\partial \mathcal{R}_{x}} c^{n-s}\left(\nabla_{1}^{\rho}, \nabla_{2}^{\rho}\right) \cdot \int_{\zeta} c^{\ell}\left(\nabla^{S}\right),
$$

where $x$ is a point in $\zeta$. Noting that the first factor in the right hand side is $d\left(v_{0}, v\right)$, we proved the lemma, in view of (5.6).

Since $\operatorname{Sch}\left(F_{0}^{(r)}, S\right)=c_{r-1}(S)$ and $\operatorname{Sch}\left(v_{0}, x\right)=1$, from Lemma 5.4, we have the following:

Theorem 5.7. Let $S$ be a non-singular component of $\operatorname{Sing}(V)$ such that $V$ satisfies the Whitney condition along $S$, then,

$$
\operatorname{Sch}\left(F^{(r)}, S\right)=\operatorname{Sch}(v, x) \cdot c_{r-1}(S) .
$$

Now we wish to obtain a formula for the virtual class analogous to the one in Theorem 5.7. First, we consider the exact sequence of vector bundles on $U_{0}$ :

$$
\left.0 \rightarrow T \rho_{0} \rightarrow T \tilde{\rho}\right|_{U_{0}} \rightarrow N_{U_{0}} \rightarrow 0 .
$$

We try to compute the Chern classes $c^{j}\left(\tau_{\rho}\right)$ of the virtual bundle $\tau_{\rho}=\left.(T \tilde{\rho}-N)\right|_{U}$ on $U$ and will see that there is a canonical lifting $c_{S}^{j}\left(\tau_{\rho}\right)$ in $H^{2 j}(U, U \backslash S)$, for $j>n-s=\operatorname{rank} T \rho_{0}$, of $c^{j}\left(\tau_{\rho}\right) \in H^{2 j}(U)$. For this, we consider the covering $\mathcal{U}$ of $\tilde{U}$ consisting of $\tilde{U}$ itself and a tubular neighborhood $\tilde{U}_{0}$ of $U_{0}$ and represent $c^{j}\left(\tau_{\tilde{\rho}}\right)$, 
$\tau_{\tilde{\rho}}=T \tilde{\rho}-N$, as a Čech-de Rham cocycle on $\mathcal{U}$ (cf. [Le1], [Su2], here we use the notation in $\underline{\mathrm{Su} 2}$, Ch.II).

Let $\nabla_{0}^{\rho}$ be a connection for $T \rho_{0}$. Let $\nabla^{N}$ be a connection for $\left.N\right|_{S}$ and take a connection $\nabla_{0}^{\tilde{\rho}}$ for $\left.T \tilde{\rho}\right|_{U_{0}}$ so that $\left(\nabla_{0}^{\rho}, \nabla_{0}^{\tilde{\rho}}, \rho_{0}^{*} \nabla^{N}\right)$ is compatible with (5.8). Let $\tilde{\nabla}^{\tilde{\rho}}$ be a connection for $T \tilde{\rho}$ on $\tilde{U}$. We set $\tilde{\nabla} \tilde{\rho} \bullet=\left(\tilde{\nabla}^{\tilde{\rho}}, \tilde{\rho}^{*} \nabla^{N}\right)$ and $\nabla_{0}^{\tilde{\rho} \bullet}=\left(\nabla_{0}^{\tilde{\rho}}, \rho_{0}^{*} \nabla^{N}\right)$. Then $c^{j}\left(\tau_{\tilde{\rho}}\right)$ is represented by a cocycle in $A^{2 j}(\mathcal{U})=A^{2 j}\left(\tilde{U}_{0}\right) \oplus A^{2 j}(\tilde{U}) \oplus A^{2 j-1}\left(\tilde{U}_{0}\right)$, where $A^{*}()$ denotes the space of differential forms on the relevant open set, given by

$$
c^{j}\left(\nabla_{\star}^{\bullet}\right)=\left(c^{j}\left(\nabla_{0}^{\tilde{\rho} \bullet}\right), c^{j}\left(\tilde{\nabla}^{\tilde{\rho} \bullet}\right), c^{j}\left(\nabla_{0}^{\tilde{\rho} \bullet}, \tilde{\nabla}^{\tilde{\rho} \bullet}\right)\right) .
$$

Note that, since $\tilde{U}_{0}$ retracts to $U_{0}$, it suffices to give forms on $U_{0}$. Since the family $\left(\nabla_{0}^{\rho}, \nabla_{0}^{\tilde{\rho}}, \rho_{0}^{*} \nabla^{N}\right)$ is compatible with (5.8), we have

$$
c^{j}\left(\nabla_{0}^{\tilde{\rho} \bullet}\right)=c^{j}\left(\nabla_{0}^{\rho}\right)
$$

which vanishes for $j>n-s$ by the rank reason. Thus, for $j>n-s$, the cocycle $c^{j}\left(\nabla_{\star}^{\bullet}\right)$ is in $A^{2 j}\left(\mathcal{U}, \tilde{U}_{0}\right)=\{0\} \oplus A^{2 j}(\tilde{U}) \oplus A^{2 j-1}\left(\tilde{U}_{0}\right)$. Since the cohomology of $A^{*}\left(\mathcal{U}, \tilde{U}_{0}\right)$ is canonically isomorphic with $H^{*}(U, U \backslash S)([\mathrm{Su} 2]$, Ch.VI, 4), this cocycle defines a class, denoted $c_{S}^{j}\left(\tau_{\rho}\right)$, in $H^{2 j}(U, U \backslash S)$, which is mapped to $c^{j}\left(\tau_{\rho}\right)$ by the canonical homomorphism $H^{2 j}(U, U \backslash S) \rightarrow H^{2 j}(U)$. The class $c_{S}^{j}\left(\tau_{\rho}\right)$ does not depend on the choices of various connections. It should be also noted that it has nothing to do with the frames we discussed earlier. Denoting by $A^{2 i}(S)$ the space of $2 i$-forms on $S$, we have the integration along the fibers of $\rho$ (Su2], Ch.II, 5) $\rho_{*}: A^{2(n-s+i)}\left(\mathcal{U}, \tilde{U}_{0}\right) \rightarrow A^{2 i}(S)$, which commutes with the differentials and induces a map on the cohomology level :

$$
\rho_{*}: H^{2(n-s+i)}(U, U \backslash S) \rightarrow H^{2 i}(S) .
$$

On the cocycle level, $\rho_{*}$ assigns to $c^{n-s+i}\left(\nabla_{\star}^{\bullet}\right), i>0$, the $2 i$-form $\alpha^{i}$ on $S$ given by

$$
\alpha^{i}=\rho_{*} c^{n-s+i}\left(\tilde{\nabla}^{\tilde{\rho} \bullet}\right)+(\partial \rho)_{*} c^{n-s+i}\left(\tilde{\nabla}^{\tilde{\rho} \bullet}, \nabla_{0}^{\tilde{\rho} \bullet}\right),
$$

where $\rho_{*}$ and $(\partial \rho)_{*}$ denote the integration along the fibers of $\left.\rho\right|_{\mathcal{R}}$ and $\left.\rho\right|_{\partial \mathcal{R}}$.

We note that, in the following formulas, the classes $\rho_{*} c_{S}^{n-s+i}\left(\tau_{\rho}\right)$ for $i=1, \ldots, k-$ 1 are involved and they do not appear when $k=1$ (i.e. $V$ is a hypersurface). We denote by []$^{i}$ the component of degree $2 i$ of the relevant cohomology class.

Theorem 5.10. With the hypotheses of 5.7, we have

$$
\begin{aligned}
\operatorname{Vir}\left(F^{(r)}, S\right)= & {\left[\left(\operatorname{Vir}(v, x) \cdot\left(c^{*}(N)-c^{k}(N)\right)+\operatorname{Sch}(v, x) \cdot c^{k}(N)\right.\right.} \\
& \left.\left.+\sum_{j=1}^{k-1} \sum_{i=1}^{j} c^{j-i}(N) \cdot \rho_{*} c_{S}^{n-s+i}\left(\tau_{\rho}\right)\right) \cdot c^{*}(N)^{-1} \cdot c^{*}(S)\right]^{\ell} \frown[S] .
\end{aligned}
$$

Proof. By Lemma 5.4, it suffices to prove the formula for the radial frame $F_{0}^{(r)}$. We consider the commutative diagram of vector bundles on $U_{0}$ with exact rows and 
columns:

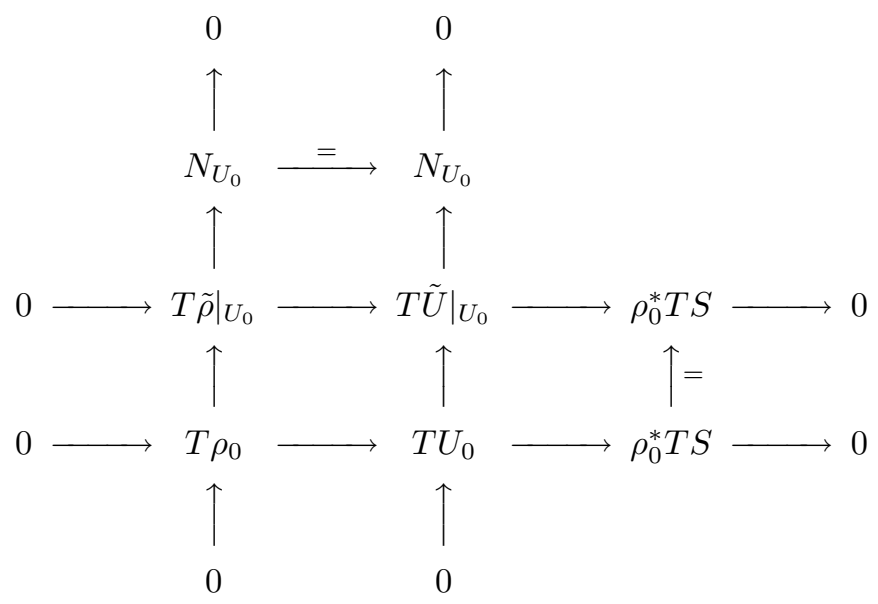

We take suitable connections that we will need. As noted before, we may assume that $v_{0}$ is tangent to the fibers of $\tilde{\rho}$ in a neighborhood $\tilde{U}_{0}^{\prime}$ of $\partial \tilde{\mathcal{R}}$. We set $U_{0}^{\prime}=\tilde{U}_{0}^{\prime} \cap V$. Let $\nabla_{0}^{\rho}$ be a $v_{0}$-trivial connection for $T \rho_{0}$ on a neighborhood $W$ of $U_{0}^{\prime} \cap D^{2 q}$ and $\nabla^{S}$ an $F_{0}^{(r-1)}$-trivial connection for $T S$ on a neighborhood of $S \cap D^{2 q}$. We take a connection $\nabla^{V}$ for $T U_{0}$ so that $\left(\nabla_{0}^{\rho}, \nabla^{V}, \rho_{0}^{*} \nabla^{S}\right)$ is compatible with the third row in (5.11). Thus $\nabla^{V}$ is $F_{0}^{(r)}$-trivial on $W$, as in the proof of Lemma 5.4. Let $\nabla^{N}$ be a connection for $\left.N\right|_{S}$ and take a connection $\nabla_{0}^{\tilde{\rho}}$ for $\left.T \tilde{\rho}\right|_{U_{0}}$ so that $\left(\nabla_{0}^{\rho}, \nabla_{0}^{\tilde{\rho}}, \rho_{0}^{*} \nabla^{N}\right)$ is compatible with the first column in (5.11). Finally take a connection $\nabla_{0}$ for $\left.T \tilde{U}\right|_{U_{0}}$ so that $\left(\nabla_{0}^{\tilde{\rho}}, \nabla_{0}, \rho_{0}^{*} \nabla^{S}\right)$ is compatible with the second row. Then $\left(\nabla^{V}, \nabla_{0}, \rho_{0}^{*} \nabla^{N}\right)$ is compatible with the second column. We may extend $\nabla_{0}^{\tilde{\rho}}$ and $\nabla_{0}$ to connections $\tilde{\nabla}_{0}^{\tilde{\rho}}$ and $\tilde{\nabla}_{0}$ for $T \tilde{\rho}$ and $T \tilde{U}$, respectively, on a neighborhood $\tilde{W}$ of $\tilde{U}_{0}^{\prime} \cap D^{2 q}$ so that $\tilde{\nabla}_{0}^{\tilde{\rho}}$ is $v_{0}$-trivial and that $\left(\tilde{\nabla}_{0}^{\tilde{\rho}}, \tilde{\nabla}_{0}, \tilde{\rho}^{*} \nabla^{S}\right)$ is compatible with the exact sequence

$$
0 \rightarrow T \tilde{\rho} \rightarrow T \tilde{U} \rightarrow \tilde{\rho}^{*} T S \rightarrow 0 .
$$

Thus $\tilde{\nabla}_{0}$ is $F_{0}^{(r)}$-trivial on $\tilde{W}$. Let $\tilde{\nabla} \tilde{\rho}$ be a connection for $T \tilde{\rho}$ on $\tilde{U}$. We take a connection $\tilde{\nabla}$ for $T \tilde{U}$ so that $\left(\tilde{\nabla} \tilde{\rho}, \tilde{\nabla}, \tilde{\rho}^{*} \nabla^{S}\right)$ is compatible with (5.12). With these connections, $\operatorname{Vir}\left(F_{0}^{(r)}, S\right)$ is given by $(4.3)$, with $\nabla^{\bullet}=\left(\tilde{\nabla}, \tilde{\rho}^{*} \nabla^{N}\right)$ and $\nabla_{0}^{\bullet}=$ $\left(\nabla_{0}, \rho_{0}^{*} \nabla^{N}\right)$.

Noting that the rank of $T \tilde{\rho}$ is $m-s=n+k-s$, from (5.12), we have

$$
\begin{aligned}
c^{p}(T \tilde{U}-N)= & \sum_{j>\ell}\left[\tilde{\rho}^{*} c^{*}(S) c^{*}(N)^{-1}\right]^{j} \cdot c^{p-j}(T \tilde{\rho}) \\
& +\sum_{i=0}^{k}\left[\tilde{\rho}^{*} c^{*}(S) c^{*}(N)^{-1}\right]^{\ell-i} \cdot c^{n-s+i}(T \tilde{\rho}) .
\end{aligned}
$$

On the other hand,

$$
c^{n-s+i}(T \tilde{\rho})=\sum_{j=0}^{i} c^{n-s+i-j}\left(\tau_{\tilde{\rho}}\right) \cdot c^{j}(N)+\sum_{j>i} c^{n-s+i-j}\left(\tau_{\tilde{\rho}}\right) \cdot c^{j}(N) .
$$


From the above two equalities, we get

$$
\begin{aligned}
c^{p}(T \tilde{U}-N)= & \sum_{i=0}^{k-1}\left[\tilde{\rho}^{*} c^{*}(S) c^{*}(N)^{-1} c^{i}(N)\right]^{\ell} \cdot c^{n-s}\left(\tau_{\tilde{\rho}}\right) \\
& +\sum_{j=1}^{k-1} \sum_{i=1}^{j}\left[\tilde{\rho}^{*} c^{*}(S) c^{*}(N)^{-1} c^{j-i}(N)\right]^{\ell-i} \cdot c^{n-s+i}\left(\tau_{\tilde{\rho}}\right) \\
& +\left[\tilde{\rho}^{*} c^{*}(S) c^{*}(N)^{-1}\right]^{\ell-k} \cdot c^{m-s}(T \tilde{\rho})+c^{p}(S, N, T \tilde{\rho}),
\end{aligned}
$$

where $c^{p}(S, N, T \tilde{\rho})$ denotes a polynomial in the Chern classes of $T S, N$ and $T \tilde{\rho}$, homogeneous of degree $p$, such that each of its monomials involves the Chern classes of $T S$ and $N$ of total degree greater than $\ell$. Hence, setting $\tilde{\nabla}^{\tilde{\rho} \bullet}=\left(\tilde{\nabla} \tilde{\rho}, \tilde{\rho}^{*} \nabla^{N}\right)$ and $\nabla_{0}^{\tilde{\rho} \bullet}=\left(\nabla_{0}^{\tilde{\rho}}, \rho_{0}^{*} \nabla^{N}\right)$, we have

$$
\begin{aligned}
c^{p}\left(\nabla^{\bullet}\right)= & \sum_{i=0}^{k-1}\left[\tilde{\rho}^{*} c^{*}\left(\nabla^{S}\right) c^{*}\left(\nabla^{N}\right)^{-1} c^{i}\left(\nabla^{N}\right)\right]^{\ell} \cdot c^{n-s}\left(\tilde{\nabla}^{\tilde{\rho} \bullet}\right) \\
& +\sum_{j=1}^{k-1} \sum_{i=1}^{j}\left[\tilde{\rho}^{*} c^{*}\left(\nabla^{S}\right) c^{*}\left(\nabla^{N}\right)^{-1} c^{j-i}\left(\nabla^{N}\right)\right]^{\ell-i} \cdot c^{n-s+i}\left(\tilde{\nabla}^{\tilde{\rho} \bullet}\right) \\
& +\left[\tilde{\rho}^{*} c^{*}\left(\nabla^{S}\right) c^{*}\left(\nabla^{N}\right)^{-1}\right]^{\ell-k} \cdot c^{m-s}\left(\tilde{\nabla}^{\tilde{\rho}}\right)+c^{p}\left(\nabla^{S}, \nabla^{N}, \tilde{\nabla}^{\tilde{\rho}}\right),
\end{aligned}
$$

and

$$
\begin{aligned}
c^{p}\left(\nabla^{\bullet}, \nabla_{0}^{\bullet}\right) & =\sum_{i=0}^{k-1}\left[\tilde{\rho}^{*} c^{*}\left(\nabla^{S}\right) c^{*}\left(\nabla^{N}\right)^{-1} c^{i}\left(\nabla^{N}\right)\right]^{\ell} \cdot c^{n-s}\left(\tilde{\nabla} \tilde{\rho} \bullet, \nabla_{0}^{\tilde{\rho} \bullet}\right) \\
& +\sum_{j=1}^{k-1} \sum_{i=1}^{j}\left[\tilde{\rho}^{*} c^{*}\left(\nabla^{S}\right) c^{*}\left(\nabla^{N}\right)^{-1} c^{j-i}\left(\nabla^{N}\right)\right]^{\ell-i} \cdot c^{n-s+i}\left(\tilde{\nabla}^{\tilde{\rho} \bullet}, \nabla_{0}^{\tilde{\rho} \bullet}\right) \\
& +\left[\tilde{\rho}^{*} c^{*}\left(\nabla^{S}\right) c^{*}\left(\nabla^{N}\right)^{-1}\right]^{\ell-k} \cdot c^{m-s}\left(\tilde{\nabla^{\tilde{\rho}}}, \nabla_{0}^{\tilde{\rho}}\right)+c^{p}\left(\nabla^{S}, \nabla^{N},\left(\tilde{\nabla} \tilde{\rho}^{\tilde{\rho}}, \nabla_{0}^{\tilde{\rho}}\right)\right) .
\end{aligned}
$$

Noting that a relative $2 q$-cycle $\gamma$ fibers over a $2 \ell$ cycle $\zeta$ in $S$, as in the proof of Theorem 5.7, and that $\left(0, c^{m-s}\left(\tilde{\nabla}^{\tilde{\rho}}\right), c^{m-s}\left(\tilde{\nabla}_{0}^{\tilde{\rho}}, \tilde{\nabla} \tilde{\rho}\right)\right)$ is a Cech-de Rham cocycle on the ambient space, by the projection formula and duality ( $\underline{\mathrm{Su} 3}$ ], Theorem 6.4 and Remark 6.6), we have

$$
\begin{aligned}
& \int_{\gamma \cap \mathcal{R}} c^{p}\left(\nabla^{\bullet}\right)+\int_{\gamma \cap \partial \mathcal{R}} c^{p}\left(\nabla^{\bullet}, \nabla_{0}^{\bullet}\right) \\
= & \left(\int_{\mathcal{R}_{x}} c^{n-s}(\tilde{\nabla} \tilde{\rho} \bullet)+\int_{\partial \mathcal{R}_{x}} c^{n-s}\left(\tilde{\nabla}^{\tilde{\rho} \bullet}, \nabla_{0}^{\tilde{\rho} \bullet}\right)\right) \cdot \sum_{i=0}^{k-1} \int_{\zeta}\left[c^{*}\left(\nabla^{S}\right) c^{*}\left(\nabla^{N}\right)^{-1} c^{i}\left(\nabla^{N}\right)\right]^{\ell} \\
& +\sum_{j=1}^{k-1} \sum_{i=1}^{j} \int_{\zeta}\left[c^{*}\left(\nabla^{S}\right) c^{*}\left(\nabla^{N}\right)^{-1} c^{j-i}\left(\nabla^{N}\right) \alpha^{i}\right]^{\ell} \\
& +\left(\int_{\tilde{\mathcal{R}}_{x}} c^{m-s}\left(\tilde{\nabla}^{\tilde{\rho}}\right)+\int_{\partial \tilde{\mathcal{R}}_{x}} c^{m-s}\left(\tilde{\nabla}^{\tilde{\rho}}, \tilde{\nabla}_{0}^{\tilde{\rho}}\right)\right) \cdot \int_{\zeta}\left[c^{*}\left(\nabla^{S}\right) c^{*}\left(\nabla^{N}\right)^{-1} c^{k}\left(\nabla^{N}\right)\right]^{\ell}
\end{aligned}
$$

where $x$ is a point in $\zeta$ and $\alpha^{i}$ is the $2 i$-form given by (5.9). Note that the integrals of $c^{p}\left(\nabla^{S}, \nabla^{N}, \tilde{\nabla}^{\tilde{\rho}}\right)$ and $c^{p}\left(\nabla^{S}, \nabla^{N},\left(\tilde{\nabla} \tilde{\rho}, \nabla_{0}^{\tilde{\rho}}\right)\right)$ vanish by dimension reason, since they involve the pull-back of forms on $S$ of degree greater than $2 \ell$. Recalling that, in the right hand side above, the first factor of the first term is $\operatorname{Vir}\left(v_{0}, x\right)$ and the 
first factor in the third term is, by Proposition 3.3, $\operatorname{Sch}\left(v_{0}, x\right)(=1)$, we proved the theorem.

From Theorems 5.7 and 5.10, we get the following Lefschetz type formula for the Milnor class.

Corollary 5.13. Let $S$ be a non-singular connected component of $\operatorname{Sing}(V)$ such that $V$ satisfies the Whitney condition along $S$. Then

$$
\begin{aligned}
\mu_{*}(V, S)= & \left((-1)^{s} \mu(V \cap H, x) \cdot\left(c^{*}(N)-c^{k}(N)\right)\right. \\
& \left.+(-1)^{n} \sum_{j=1}^{k-1} \sum_{i=1}^{j} c^{j-i}(N) \cdot \rho_{*} c_{S}^{n-s+i}\left(\tau_{\rho}\right)\right) \cdot c^{*}(N)^{-1} \cdot c^{*}(S) \frown[S],
\end{aligned}
$$

where $H$ denotes an $(m-s)$-dimensional plane transverse to $S$ in $M$. In particular, when $k=1$,

$$
\mu_{*}(V, S)=(-1)^{s} \mu(V \cap H, x) \cdot c^{*}(N)^{-1} \cdot c^{*}(S) \frown[S] .
$$

Also, for arbitrary $k$,

$$
\mu_{s}(V, S)=(-1)^{s} \mu(V \cap H, x) \cdot[S] .
$$

Remark 5.14. As is seen from the above proofs, Theorems 5.7 and 5.10 hold under an assumption weaker than the Whitney condition. Namely, we only need that there is a Whitney stratification of $M$ compatible with $V$ and $S$ such that the $2(s-r+1)$ skeleton $S \cap D^{2 q}$ of $S$ is in the top dimensional stratum of $S$. Accordingly, under this assumption, we have a formula for $\mu_{r-1}(V, S)$ taking the terms of corresponding dimension in 5.13. This will be used in Example 7.2.

Remark 5.15. In the previous version of this paper (Hokkaido University Preprint Series in Mathematics No. 413, May 1998), we considered the forms $\omega^{i}$ on $S$ given by $\omega^{i}=\rho_{*} c^{n-s+i}\left(\tilde{\nabla}_{0}^{\tilde{\rho}}\right)+(\partial \rho)_{*} c^{n-s+i}\left(\tilde{\nabla}_{0}^{\tilde{\rho}}, \nabla^{\tilde{\rho}}\right)$. They are related to the $\alpha^{i}$ 's by

$$
\omega^{i}=\operatorname{Vir}(v, x) \cdot c^{i}\left(\nabla^{N}\right)+\sum_{j=1}^{i} c^{i-j}\left(\nabla^{N}\right) \alpha^{j} .
$$

In particular, each $\omega^{i}$ is a closed form on $S$. See [OY for more properties of $\omega^{i}$.

\section{Generalized Milnor number}

As in the previous sections, let $V \subset M$ be defined by a holomorphic section of a vector bundle of rank $k$ and let $S$ be a connected component of $\operatorname{Sing}(V)$.

Definition 6.1. The generalized Milnor number $\mu(V, S)$ of $V$ at $S$ is defined as

$$
\mu(V, S)=(-1)^{n+1}(\operatorname{Sch}(v, S)-\operatorname{Vir}(v, S)),
$$

where $v$ is a vector field on a neighborhood $U$ of $S$ in $V$, non-singular on $U \backslash S$.

This definition does not depend on the choice of the vector field $v$ and is equal to $\mu_{0}(V, S)$ in Definition 5.1. If $(V, a)$ is an isolated complete intersection singularity germ, for a radial vector field $v_{0}, \operatorname{Sch}\left(v_{0}, a\right)=1$ and $\operatorname{Vir}\left(v_{0}, a\right)=\chi(F)$, where $F$ denotes the Milnor fiber. Thus the above Milnor number coincides with the usual one in [Mi], [H], Lo].

We recall that the classical Milnor number of an isolated singular point $\mathrm{Mi}$ has been generalized to the case of non-isolated hypersurface singularities by A. 
Parusiński $[\mathrm{P}$ in the following way. Recall that a hypersurface $V$ in $M$ is always defined by a holomorphic section $s$ of a line bundle $N$ over $M$. There is a canonical vector bundle homomorphism $\pi:\left.\left.T M\right|_{V} \rightarrow N\right|_{V}$ which extends the one in (4.1). Note that $\operatorname{Sing}(V)$ coincides with the set of points in $V$ where $\pi$ fails to be surjective. Now let $\nabla^{\prime}$ be a connection for $N$ of type $(1,0)$. This means that in the decomposition $\nabla^{\prime}=\nabla^{(1,0)}+\nabla^{(0,1)}$ of $\nabla^{\prime}$ into the $(1,0)$ and $(0,1)$ components, we have $\nabla^{(0,1)}=\bar{\partial}$. Since $s$ is holomorphic, we have $\nabla^{\prime} s=\nabla^{(1,0)} s$, which is a $C^{\infty}$ section $t$ of $T^{*} M \otimes N$. Write $\tilde{\pi}: T M \rightarrow N$ the corresponding bundle homomorphism. Let $S$ be a compact component of $\operatorname{Sing}(V)$ and $\tilde{U}$ a neighborhood of $S$ in $M$ disjoint from the other components. It is shown in $[\mathrm{P}]$ that $S$ coincides with a connected component of the zero set of $t$. Then Parusinski defines the Milnor number $\mu_{S}(V)$ to be the intersection number in $\tilde{U}$ of the section $t$ of $T^{*} M \otimes N$ with the zero section.

Theorem 6.2. For a hypersurface $V$, we have

$$
\mu_{S}(V)=\mu(V, S)
$$

Proof. First we give an integral formula for $\mu_{S}(V)$, similar to (3.2) for the bundle $T^{*} M \otimes N$. Let $\tilde{\mathcal{R}}$ be a $\left(D^{\prime}\right)$-cellular tube around $S$ in $\tilde{U}$ and $v_{0}$ a radial vector field near $S$ pointing outward everywhere on $\partial \tilde{\mathcal{R}}$. Moreover, let $\nabla$ be an arbitrary connection for $T M$ on $\tilde{U}$ and $\nabla^{\bullet}=\left(\nabla, \nabla^{\prime}\right)$ the corresponding pair of connections on $\tilde{U}$. There exists a $v_{0}$-trivial connection $\nabla_{0}$ for $T M$ on $\tilde{U} \backslash S$, such that the pair $\nabla_{0}^{\bullet}=\left(\nabla_{0}, \nabla^{\prime}\right)$ is compatible, i.e., such that $\nabla^{\prime} \circ \tilde{\pi}=\tilde{\pi} \circ \nabla_{0}$; furthermore, the restriction of $\nabla_{0}^{\bullet}$ to $V_{0}$ is compatible. Denote by $D$ the connection $\nabla^{*} \otimes \nabla^{\prime}$ for $T^{*} M \otimes N$ on $\tilde{U}$ and by $D_{0}$ the connection $\nabla_{0}^{*} \otimes \nabla^{\prime}$ for $T^{*} M \otimes N$ on $\tilde{U} \backslash S$. Since the pair $\nabla_{0}^{\bullet}$ is compatible, the connection $D_{0}$ is $t$-trivial. With these, we have

$$
\mu_{S}(V)=\int_{\tilde{\mathcal{R}}} c^{n+1}(D)+\int_{\partial \tilde{\mathcal{R}}} c^{n+1}\left(D, D_{0}\right)
$$

We compute

$$
c^{n+1}(D)=(-1)^{n}\left(c^{1}\left(\nabla^{\prime}\right) \cdot c^{n}\left(\nabla^{\bullet}\right)-c^{n+1}(\nabla)\right),
$$

and

$$
c^{n+1}\left(D, D_{0}\right)=(-1)^{n}\left(c^{1}\left(\nabla^{\prime}\right) \cdot c^{n}\left(\nabla^{\bullet}, \nabla_{0}^{\bullet}\right)-c^{n+1}\left(\nabla, \nabla_{0}\right)\right) .
$$

By duality ([Su3], Theorem 6.4), we have

$$
\begin{aligned}
\int_{\tilde{\mathcal{R}}} c^{1}\left(\nabla^{\prime}\right) \cdot c^{n}\left(\nabla^{\bullet}\right) & +\int_{\partial \tilde{\mathcal{R}}} c^{1}\left(\nabla^{\prime}\right) \cdot c^{n}\left(\nabla^{\bullet}, \nabla_{0}^{\bullet}\right) \\
& =\int_{\mathcal{R}} c^{n}\left(\nabla^{\bullet}\right)+\int_{\partial \mathcal{R}} c^{n}\left(\nabla^{\bullet}, \nabla_{0}^{\bullet}\right)=\operatorname{Vir}\left(v_{0}, S\right) .
\end{aligned}
$$

On the other hand,

$$
\int_{\tilde{\mathcal{R}}} c^{n+1}(\nabla)+\int_{\partial \tilde{\mathcal{R}}} c^{n+1}\left(\nabla, \nabla_{0}\right)=\chi(\tilde{\mathcal{R}})=\chi(S)=\operatorname{Sch}\left(v_{0}, S\right),
$$

which proves the theorem. 


\section{EXAMPLES}

Let $M$ be the complex projective space $\mathbb{C P}^{m}$ and $L \rightarrow M$ the hyperplane bundle (dual of the tautological bundle). Then $T M$ is stably equivalent to $(m+1) L$. Furthermore if $V$ is the intersection of $k$ hypersurfaces in $M$, each of which being defined by a homogeneous polynomial of degree $d_{j}, 1 \leq j \leq k$, then $N=\bigoplus_{j=1}^{k} L^{d_{j}}$. Thus, if we set $u=c^{1}(L)$, the total Chern class of $T M-N$ is given by

$$
c^{*}(T M-N)=\frac{(1+u)^{m+1}}{\prod_{j}\left(1+d_{j} u\right)} .
$$

Example 7.2. Let $M=\mathbb{C P}^{3}$, with homogeneous coordinates $[X, Y, Z, T]$, and let $\Gamma$ be the curve defined by $X^{2}-Y T=0$ and $Z^{2}-X Y=0$. The only singular point of $\Gamma$ is the point $p_{0}=[0,0,0,1]$.

Let us consider the vector field $v$ defined in the affine space $T \neq 0$ by $v=$ $2 x \frac{\partial}{\partial x}+4 y \frac{\partial}{\partial y}+3 z \frac{\partial}{\partial z}$, with respect to the affine coordinates $(x, y, z)=\left(\frac{X}{T}, \frac{Y}{T}, \frac{Z}{T}\right)$. The vector field $v$ is tangent to $\Gamma \backslash\left\{p_{0}\right\}$, radial outbound from $p_{0}$ and it has another singular point $p=[0,1,0,0]$ on $\Gamma$ where it is also radial. We have $\operatorname{Sch}\left(v, p_{0}\right)=1$ and $\operatorname{Sch}(v, p)=1$ and $\chi(\Gamma)=c_{0}(\Gamma)=2$ by $\underline{\operatorname{Sc} 2}$.

On the other hand, the formula of [LSS], p. 186, gives $\operatorname{Vir}\left(v, p_{0}\right)=-1$, therefore $\mu_{0}\left(\Gamma, p_{0}\right)=2$ by definition. This implies that the virtual class $c^{1}\left(\tau_{\Gamma}\right)=$ $c^{1}\left(\left.(T M-N)\right|_{\Gamma}\right)$ is 0 , noting that $\operatorname{Vir}(v, p)=1$ as $p$ is a regular point of $\Gamma$. This last formula can be also computed directly from (7.1).

Example 7.3. Let $M=\mathbb{C P}^{4}$, with homogeneous coordinates $[X, Y, Z, T, U]$, and let $V$ be the cone over the curve $\Gamma$ of Example 7.2, i.e., the surface defined by $X^{2}-Y T=0$ and $Z^{2}-X Y=0$. If we set $p_{0}=[0,0,0,1,0]$ and $q=[0,0,0,0,1]$, then the singular set of $V$ is the line $S$ through $p_{0}$ and $q$. Let us consider the Whitney stratification of $M$ consisting of $\{q\}, S \backslash\{q\}, V \backslash S$ and $M \backslash V$.

The vector fields defined in the affine space $U \neq 0$ by

$$
v_{1}=2 x \frac{\partial}{\partial x}+4 y \frac{\partial}{\partial y}+3 z \frac{\partial}{\partial z} \quad \text { and } \quad v_{2}=x \frac{\partial}{\partial x}+y \frac{\partial}{\partial y}+z \frac{\partial}{\partial z}+t \frac{\partial}{\partial t},
$$

with respect to the affine coordinates $(x, y, z, t)=\left(\frac{X}{U}, \frac{Y}{U}, \frac{Z}{U}, \frac{T}{U}\right)$, extend naturally to the hyperplane at infinity $U=0$ and they are stratified vector fields on $V$. We define $w_{a}=v_{1}+a v_{2}$ for a complex number $a \neq-2,-3,-4$. The singular set of $w_{a}$ consists of $S$ and the point $p=[0,1,0,0,0]$ and $w_{a}$ is radial outbound from $p$ and $S$.

Since the singular set $S$ is one dimensional, there are two Milnor classes $\mu_{0}(V, S)$ and $\mu_{1}(V, S)$. We have $\operatorname{Sch}\left(w_{a}, S\right)=\chi(S)=2$ and $\operatorname{Sch}\left(w_{a}, p\right)=1$, hence $\chi(V)=$ $\chi(S)+1=3$. On the other hand, by $(7.1)$ we have $c^{2}\left(\tau_{V}\right) \frown[V]=8$. Since the point $p$ is regular, $\operatorname{Vir}\left(w_{a}, p\right)=\operatorname{Sch}\left(w_{a}, p\right)=1$. Thus $\operatorname{Vir}\left(w_{a}, S\right)=8-1=7$ and $\mu_{0}(V, S)=\operatorname{Vir}\left(w_{a}, S\right)-\operatorname{Sch}\left(w_{a}, S\right)=5$.

The Milnor class $\mu_{1}(V, S)$ can be computed using Corollary 5.13 (see also Remark 5.14). In this case, $s=r-1$ and, with the above stratification, the 0-skeleton (of the dual cellular decomposition) of $S$ is in the top-dimensional stratum $S \backslash\{q\}$ of $S$. Hence we have

$$
\mu_{1}(V, S)=-\mu_{0}\left(\Gamma, p_{0}\right) \cdot[S] .
$$

By Example 7.2, $\mu_{0}\left(\Gamma, p_{0}\right)=2$, thus $\mu_{1}(V, S)=-2[S]$. 


\section{REFERENCES}

[A1] P. Aluffi, Singular schemes of hypersurfaces, Duke Math. J. 80 (1995), 325-351. MR 97b:14057

[A2] P. Aluffi, Chern classes for singular hypersurfaces, Trans. Amer. Math. Soc. 351 (1999), 3989-4026. CMP 99:15

[BB] P. Baum and R. Bott, Singularities of holomorphic foliations, J. Differential Geom. 7 (1972), 279-342. MR 51:14092

[Bo] R. Bott, Lectures on characteristic classes and foliations, Lectures on Algebraic and Differential Topology, Lecture Notes in Mathematics 279, Springer-Verlag, New York, Heidelberg, Berlin, 1972, pp. 1-94. MR 50:14777

[Br1] J.-P. Brasselet, Définition combinatoire des homomorphismes de Poincaré, Alexander et Thom pour une pseudo-variété, Caractéristique d'Euler-Poincaré, Astérisque 82-83, Société Mathématique de France, 1981, pp. 71-91. MR 83d:57014

[Br2] J.-P. Brasselet, From Chern classes to Milnor classes, Singularities - Sapporo 1998, Advanced Studies in Pure Math. 29, Math. Soc. Japan, 2000, pp. 31-52. CMP 2001:11

[BS] J.-P. Brasselet et M.-H. Schwartz, Sur les classes de Chern d'un ensemble analytique complexe, Caractéristique d'Euler-Poincaré, Astérisque 82-83, Société Mathématique de France, 1981, pp. 93-147. MR 83h:32011

[F] W. Fulton, Intersection Theory, Springer-Verlag, Berlin, Heidelberg, New York, Tokyo, 1984. MR 85k:14004

[FJ] W. Fulton and K. Johnson, Canonical classes on singular varieties, Manuscripta Math. 32 (1980), 381-389. MR 82c:14017

[GSV] X. Gómez-Mont, J. Seade and A. Verjovsky, The index of a holomorphic flow with an isolated singularity, Math. Ann. 291 (1991), 737-751. MR 93d:32066

[H] H. Hamm, Lokale topologische Eigenschaften komplexer Räume, Math. Ann. 191 (1971), 235-252. MR 44:3357

[Le1] D. Lehmann, Variétés stratifiées $C^{\infty}$ : Intégration de Čech-de Rham et théorie de ChernWeil, Geometry and Topology of Submanifolds II, Proc. Conf., May 30-June 3, 1988, Avignon, France, World Scientific, Singapore, 1990, pp. 205-248. MR 92a:58007

[Le2] D. Lehmann, A Chern-Weil theory for Milnor classes, Singularities - Sapporo 1998, Advanced Studies in Pure Math. 29, Math. Soc. Japan, 2000, pp. 181-201. CMP 2001:11

[LSS] D. Lehmann, M. Soares and T. Suwa, On the index of a holomorphic vector field tangent to a singular variety, Bol. Soc. Bras. Mat. 26 (1995), 183-199. MR 96k:32080

[Lo] E. Looijenga, Isolated Singular Points on Complete Intersections, London Mathematical Society Lecture Note Series 77, Cambridge Univ. Press, 1984. MR 86a:32021

[Ma] R. MacPherson, Chern classes for singular algebraic varieties, Ann. of Math. 100 (1974), 423-432. MR 50:13587

[Mi] J. Milnor, Singular Points of Complex Hypersurfaces, Annals of Mathematics Studies 61, Princeton University Press, Princeton, 1968. MR 39:969

[OY] T. Ohmoto and S. Yokura, Product formula for the Milnor class, to appear in Bull. Polish Acad. Sci. 48 (2000), 387-401. CMP 2001:05

[P] A. Parusiński, A generalization of the Milnor number, Math. Ann. 281 (1988), 247-254. MR 89k:32023

[PP1] A. Parusiński and P. Pragacz, A formula for the Euler characteristic of singular hypersurfaces, J. Algebraic Geom. 4 (1995), 337-351. MR 96i:32039

[PP2] A. Parusiński and P. Pragacz, Characteristic classes of hypersurfaces and characteristic cycles, J. Algebraic Geom. 10 (2001), 63-79. CMP 2001:04

[Sc1] M.-H. Schwartz, Classes caractéristiques définies par une stratification d'une variété analytique complexe, C.R. Acad. Sci. Paris 260 (1965), 3262-3264, 3535-3537. MR 35:3707. MR 32:1727

[Sc2] M.-H. Schwartz, Champs radiaux sur une stratification analytique complexe, Travaux en cours 39, Hermann, Paris, 1991. MR 92i:32041

[Sc3] M.-H. Schwartz, Classes de Chern des ensembles analytiques, Actualités Mathématiques, Hermann, Paris, 2000.

[SS1] J. Seade and T. Suwa, A residue formula for the index of a holomorphic flow, Math. Ann. 304 (1996), 621-634. MR 97j:32034

[SS2] J. Seade and T. Suwa, An adjunction formula for local complete intersections, International J. Math. 9 (1998), 759-768. MR 99k:32059 
[Su1] T. Suwa, Classes de Chern des intersections complètes locales, C.R. Acad. Sci. Paris 324 (1996), 67-70. MR 97m:14003

[Su2] T. Suwa, Indices of Vector Fields and Residues of Singular Holomorphic Foliations, Actualités Mathématiques, Hermann, Paris, 1998. MR 99h:32042

[Su3] T. Suwa, Dual class of a subvariety, Tokyo J. Math. 23 (2000), 51-68. MR 2001e:32048

[St] N. Steenrod, The Topology of Fibre Bundles, Princeton Univ. Press, Princeton, 1951. MR 12:522b

[W] H. Whitney, Tangents to an analytic variety, Ann. of Math. 81 (1965), 496-549. MR 33:745

[Y] S. Yokura, On a Milnor class, Preprint 1997.

Institut de Mathématiques de Luminy, UPR 9016 CNRS, Campus de Luminy - Case 907, 13288 Marseille Cedex 9, France

E-mail address: jpb@iml.univ-mrs.fr

Département des Sciences Mathématiques, Université de Montpellier iI, 34095 Montpellier Cedex 5, France

E-mail address: lehmann@darboux.math.univ-montp2.fr

Instituto de Matemáticas, Universidad Nacional Autónoma de México, Ciudad Universitaria, Circuito Exterior, México 04510 D.F., México

E-mail address: jseade@matem.unam.mx

Department of Mathematics, Hokkaido University, Sapporo 060-0810, Japan

E-mail address: suwa@math.sci.hokudai.ac.jp 